Author's post-print published in Brain Research Vol. 1594 , pp. 136-153, 2015 http://dx.doi.org/10.1016/j.brainres.2014.10.054

\title{
Modulation of functional connectivity following visual adaptation: homeostasis in V1
}

\section{BACHATENE ${ }^{\mathrm{a}, \mathrm{b}}$, V. BHARMAURIA ${ }^{\mathrm{a}, \mathrm{b}}$, S. CATTAN ${ }^{\mathrm{a}, \mathrm{b}}$, J. ROUAT ${ }^{\mathrm{a}, \mathrm{b}}$ AND S. MOLOTCHNIKOFF ${ }^{a, b *}$}

${ }^{a}$ Laboratoire de Neurosciences de la Vision, Département de Sciences Biologiques, Université de Montréal, Montréal, QC, Canada

${ }^{\mathrm{b}}$ Neurosciences Computationnelles et Traitement Intelligent des Signaux (NECOTIS, Université de Sherbrooke, Québec, Canada)

*Corresponding author. Address: Department of Biological Sciences,

University of Montreal, C.P. 6128 , succursale

-ville, Montréal,

QC H3C 3J7, Canada. Tel: +1-514-343-6616; fax: +1-514-343-2293.

E-mail address: stephane.molotchnikoff@umontreal.ca (S. Molotchnikoff).

Abbreviations: CCG, crosscorrelogram; OSI, orientation selectivity index; RF, receptive field; TC, trial-count correlation; V1, primary visual cortex.

Abstract - Sensory neurons exhibit remarkable adaptability in acquiring new optimal selectivity to unfamiliar features when a new stimulus becomes prevalent in the environment. In conventionally prepared adult anesthetized cats, we used visual adaptation to change the preferred orientation selectivity in V1 neurons. Cortical circuits are dominated by complex and intricate connections between neurons. Cross-correlation of cellular spike-trains discloses the putative functional connection between two neurons. We sought to investigate changes in these links following a twelve minute uninterrupted application of a specific, usually non-preferred, orientation. We report that visual adaptation, mimicking training, modulates the magnitude of cross-correlograms suggesting that the strength of inter-neuronal relationships is modified. While individual cell-pairs exhibit changes in their response correlation strength, the average correlation of the recorded cell cluster remains unchanged. Hence, visual adaptation induces plastic changes that impact the connectivity between neurons. 
Key words: cortical plasticity, crosscorrelation, functional connectivity, visual adaptation, neuronal network, homeostasis.

\section{Introduction}

The brain has a remarkable capacity for plastic modifications where cortical areas are able to switch to a new selection-preference in relation to the stimulus. For instance, V1 neurons are selective for stimuli features such as orientation, spatial frequency, direction and speed (Dragoi et al., 2000; Hubel and Wiesel, 1959; Hubel and Wiesel, 1968; Kohn and Movshon, 2004; Marshansky et al., 2011; Movshon, 1975), but can modify their selectivity in order to functionally reorganize the visual cortex as an « adapted cortex » to altered stimulation. Primary visual neurons are organized into cortical domains exhibiting specific connections (Hubel and Wiesel, 1959; Hubel and Wiesel, 1968; Stratford et al., 1996; Yoshimura et al., 2000).

Visual training impinges upon the spiking activity of neuronal populations (Bachatene et al., 2012; Bachatene et al., 2013; Dragoi et al., 2000; Ghisovan et al., 2009; Kohn and Movshon, 2004; Patterson et al., 2013), hence it may influence the dynamic modulation of functional relationships between cells. Indeed, the primary visual cortex recalibrates its inputs to reconfigure information processing which results in modified neuronal connectivity after plasticity phenomenon (Fahle, 2004).

Many studies have shown that neuronal connectivity is related to the stimuli preference of neurons; cellular projections are higher between neurons sharing similar preferred features (Ko et al., 2011; Stepanyants et al., 2008). For example, cells exhibiting similar orientation selectivity are grouped into orientation columns (Alloway and Roy, 2002; Bartho et al., 2004; Csicsvari et al., 1998; Hubel and Wiesel, 1959; Hubel and Wiesel, 1968; Yoshimura et al., 2005). Following cortical plasticity, the neurons change their selectivity (Bachatene et al., 2012; Dragoi et al., 2000; Ghisovan et al., 
2009) and the cortex is reorganized (Dragoi et al., 2000; Godde et al., 2002), however, the mechanisms conferring a novel circuitry on cortical neurons are as yet unknown. Here, we seek to examine the impact of adaptation on connectivity between V1 neurons. Crosscorrelation computations signify inter-neuronal relationships that are indicative of functional connections emerging from coordinated neural activities of involved neurons (Bartho et al., 2004; Denman and Contreras, 2013; Fujisawa et al., 2008; Hata et al., 1991; Konig et al., 1995; Perkel et al., 1967). Moreover, crosscorrelograms (CCG) calculate a peak-strength which yields an index of connectivity between neurons (Alloway and Roy, 2002; Wise et al., 2010). Significant peaks within a short time-window in the CCG's are indicative of putative functional linkages between units (Bartho et al., 2004; Denman and Contreras, 2013; Fujisawa et al., 2008; Kara and Reid, 2003; Perkel et al., 1967). In the present investigation, we used crosscorrelation analyses to examine connection probabilities between V1 neuronal pairs sharing similar pre- and post-adaptation orientation-selectivity in order to reveal plastic changes in dynamic functional connectivity. In other words, how is the neural coding altered after experience-dependent plasticity? We report that visual adaptation considerably modulates the peaks-strengths among individual neuron-pairs, however, on average, peaks-strengths are not significantly different pre- and post-adaptation. Such adjustments involving cellpairs in V1 while preserving the global time relationships between neuronal spikes allow constancy within neuronal properties of an assembly of cells and at the same time adaptability to visual stimuli modifications (Benucci et al., 2013).

\section{Results}

We measured extracellular spiking activity of V1 neurons in response to visual orientation gratings after spike sorting protocol within layers II/III. The current investigation was focused on how visual adaptation (prolonged presentation of a non- 
preferred orientation stimulus within the receptive field of neurons) affects this functional dynamic. In total, 166 cells were recorded, and 105 pairs were selected based on the similarity of orientation-preference pre- and post-adaptation.

\subsection{Peaks-strengths pre- and post-adaptation: a typical cell-pair example}

The computations in Fig. 1 display a typical example of CCG analysis for a cell-pair (both cells exhibited $\pm 22^{\circ}$ similar pre- and post-adaptation selectivity). We first isolated single units from multi-unit spiking activity (see methods). Fig. 1A illustrates two simultaneously recorded cells sorted by spike wave-shapes (top), cluster distribution derived from principal component analysis (below) and autocorrelograms (right), the latter showing an absence of event (refractory period) at 0 on the time-scale.

The raster plots and peri-stimulus time histograms are shown in Fig. 1B. The inserted histograms (on the right) display the push-pull effect at both orientations of interest, the spiking activity at the initial optimal orientation declines post-adaptation, and the firing rate at the new preferred orientation (evoking weak responses pre-adaptation) increases after visual adaptation. This is demonstrated by the respective tuning curves for both neurons (Fig. 1C). The raw orientation tuning curves revealed that both cells had $\pm 22^{\circ}$ similar preferred orientation (pink neuron: $3.9 \pm 2.06 \mathrm{~Hz}$ at $67.5^{\circ}$, blue neuron: $3.19 \pm$ $1.21 \mathrm{~Hz}$ at $90^{\circ}$ ). After 12 minutes of visual adaptation to a non-preferred orientation (indicated by the downward black arrows), both tuning curves shifted their peaks to $157.5^{\circ}$ and $0^{\circ}$ for pink and blue neurons, respectively (pink neuron: $3.7 \pm 1.65 \mathrm{~Hz}$ at $157^{\circ}$, blue neuron: $1.6 \pm 0.94 \mathrm{~Hz}$ at $0^{\circ}$ ). Thereafter, shift-corrected CCG's were computed to reveal the dynamic functional links between cell-pairs (see methods for details). Fig. 1D represents the CCG's analysis for neurons shown in Fig. 1A at similar original and novel preferred orientations (pre-adaptation condition is displayed on the left and post-adaptation on the right). In this example, neuron 1 (pink unit) was set as the 
reference neuron and neuron 2 (blue unit) as the target unit. The peaks-strengths at the original optimal orientation decreased from 0.040 to 0.015 after adaptation with a large decorrelation (see also next section for general distribution). However, for the new optimal orientation, we observed a decrease from 0.040 to 0.034 and the correlation was maintained (significant peak). This reflects the dynamic functional linkage due to visual adaptation. It is to be mentioned that the aim of this study was to investigate direct functional relationships between specific neurons. Therefore, the analyses were focused on significant peaks offset from zero mark in the CCG, such analysis is a useful method to interpret the putative functional links between neurons (Bartho et al., 2004; Csicsvari et al., 1998; Denman and Contreras, 2013; Fujisawa et al., 2008).

\subsection{Adaptation influences the correlation between units}

The next figure illustrates on a global scale the changing strength of connectivity (as reflected from the peaks-strengths) for all neuronal pairs sharing the same pre- and postadaptation orientation-selectivity. Fig. 2A shows, on a cell-pair basis, the correlation of the peaks-strengths for the highest bins $(>95 \%$, time-window $\pm 5 \mathrm{~ms})$ in the CCG's prior and post-adaptation, at original optimal orientation (left graph) and new optimal orientation (right graph). For the original optimal orientation, we obtained 87 cases of significant CCG's (peaks above $95 \%$ of the confidence line) before and/or after adaptation. At the new optimal orientation, 75 significant cases were obtained. Thereafter, we correlated the peaks-strengths of neuronal pairs pre- and post-adaptation for both orientations (original and new optimal). The resultant linear regression analysis is shown in Fig. 2A. The dotted line in each graph represents the best-fit 45 degree line passing through the origin. The peak-strength before adaptation is indicated on the $\mathrm{X}$ axis, whereas the Y-axis shows the peak-strength following adaptation. The solid black line in each graph corresponds to the linear regression trend with the value of the 
coefficient of determination $\left(\mathrm{R}^{2}\right) . \mathrm{R}^{2}$ equals 0.17 and $8.10^{-5}$ for the original optimal orientation (left) and the new optimal orientation (right), respectively. Such a wide distribution of dots points to the fact that adaptation redistributes (decrease or increase of the peaks-strengths) the time-relationship between spiking activities of neuronal pairs, even though the cells are sharing the same orientation-selectivity following adaptation. We suggest that adaptation disturbs the original inter-neuronal relationships, thereby either erasing or establishing new functional connections to frame novel connectomes as a result of the emergence of newly acquired firing patterns of involved neurons.

The number of cases for diminution and augmentation of the peaks-strengths are as follows: at original optimal orientation, we found 49 cases $(56 \%)$ of diminution and 38 cases $(44 \%)$ of augmentation $(n=87)$, whereas, for new optimal orientation, a decrease was observed in 45 cases $(60 \%)$, versus 30 cases $(40 \%)$ of increase $(n=75)($ see next section for details).

Fig. 2B represents the total distribution of the peaks-strengths for all cell-pairs pre(green) and post-adaptation (orange) at the original optimal orientation (left) and the newly acquired orientation (right). Below are shown the respective non-linear regression curves with the relative coefficients of determination (Original optimal: $\mathrm{R}^{2}$ control $=0.99$, $\mathrm{R}^{2}$ post-adaptation $=0.99$, New optimal: $\mathrm{R}^{2}$ control $=0.97, \mathrm{R}^{2}$ post-adaptation $=0.97$ ).

In spite of the decorrelation following adaptation on cell-pair basis as evident from Fig. 2A, we note that in Fig. 2B, the very close superposition of the cumulative distribution of dots and curves indicates an absence of difference in global modification of the peaksstrengths in both conditions (control and post-adaptation) at each orientation of interest (original optimal and new optimal), thus suggesting that even though functional connections may change at the cell-pair basis, it is, however, likely on a populational scale that there is an inherent stability (homeostasis) to the network. Furthermore, we compared the functional connectivity between neurons sharing a $\pm 22^{\circ}$ orientation- 
preference-range and between differently tuned neurons (superior to $22^{\circ}$ of orientationpreference-range, Fig. 2C). We found a significant difference between these two groups (two-tailed t-test, $p<0.05$ ). These results suggest that even though visual adaptation modifies the peaks-strengths of crosscorrelograms that may reflect the functional connections between neurons, cells that exhibit newly similar orientations remain more strongly connected in comparison to differently tuned cells. In addition, we calculated the peaks-strengths at the adapting orientation. Overall, the modulations of the peaksstrengths set the latter at about the same average values (Fig. 2D).

\subsection{Modulation of peak-strengths}

Fig. 3 shows the population analysis of the peaks-strengths for both orientations of interest (original optimal and new optimal). We averaged the peaks-strengths for all neuronal pairs in pre-adaptation condition, and thereafter, the increase and decrease postadaptation. Fig. 3A displays all cellular pairs showing a decline of the peaks; the mean probabilities pre- and post-adaptation were 0.029 and 0.011 respectively for the original optimal orientation ( $\mathrm{n}=49$, left, significant decrease of 0.018 , two-tailed t-test, $p<0.05)$. The same tendency is observed for the new optimal orientation $(\mathrm{n}=45$, right), i.e., a significant decline of 0.019 from 0.032 to 0.013 (paired two-tailed t-test, $p<0.05$ ). Fig. 3B displays the group of pairs reacting with an increase in probability following adaptation (significant two-tailed paired t-tests for augmentation of the peaks-strengths for both original and new optimal orientations). Interestingly, the enhancement of the peak-strength for the new optimal orientation (right) is superior to the enhancement of the peak for the original optimal orientation (left); these increases are 0.027 and 0.015 respectively (One-tailed unpaired t-test, $p<0.05$ ). This is the highest modulation for this group of cells that acquire a new optimal orientation. 
These sets of data and particularly the regression analysis (Figs. 2, 3) suggest that even after visual adaptation, on average, the peaks-strengths are altered, yet, the constant application of the grating influences the time correlation of individual pairs of neurons in a flexible fashion. These computations suggest a lack of uniformity which, in turn, points toward the fact that adaptation adjusts the functional relationship between involved neurons. We suggest that such versatility in inter-neuronal relationships promotes flexibility, and, consequently, plasticity. To test whether these variations were due to spontaneous fluctuations of firing, peaks-strengths were compared for original orientations prior to, post-adaptation, and subsequently one hour after adaptation (recovery). An example of crosscorrelograms between two cells is illustrated in Fig. 3C (left). It shows the effect of adaptation on the correlation between spike trains of these two neurons and the recovery of the magnitude level one hour after adaptation. The maximal peaks of CCG's in this example were $0.06,0.013$ and 0.061 for control, postadaptation and recovery, respectively. Other examples in Fig. 3C (right, upper part) show the variability of correlation due to adaptation process as the peak strength values return to control levels after recovery. These examples indicate that visual adaptation modulates the magnitude of the peak strength between correlated neuronal spike trains.

Globally, the average values were $0.022 \pm 0.003,0.019 \pm 0.002,0.022 \pm 0.003$ for control, post-adaptation and recovery, respectively (Fig. 3C right, lower part, random sample, $\mathrm{n}=41)$.

Additionally, we compared the CCG-magnitudes between the stimulus-condition and the non-stimulus-condition (spontaneous activity of the recorded neurons), and found a significant difference (two-tailed t-test, $p<0.05$ ). Crosscorrelogram-magnitudes during spontaneous activity were very low, suggesting that connectivity-strength is due to the presentation of stimulus rather than spontaneous neuronal firing (Fig. 3D). Moreover, stimulation was repeated in order to compare the CCG-values at different times. Fig. 3E 
shows an example of two CCG's between two neurons at time $t_{1}$ (attempt 1) and $t_{2}$ (attempt 2); the time-laps between both attempts was one hour. The values of the CCGpeaks were 0.040 and 0.039 , respectively. Taken together, these results demonstrate that the CCG's remain constant.

\subsection{Dynamics of correlation}

Firing rate of neurons pre- and post- visual adaptation is changed mostly for two main orientation classes: the original optimal orientation and the new acquired optimal orientation. It is changed in such a way that we generally notice a decline of evoked firing activity for the original preferred stimulus and an increase of elicited discharges for the new preferred stimulus by an already described push-pull mechanism, therefore leading to the observed shifts after the adaptation process (Bachatene et al., 2012; Bachatene et al., 2013; Ghisovan et al., 2009). We thus calculated a Fano Factor $\left(\mathrm{F}=\sigma^{2} /\right.$ $\mu)$ by dividing the variance $\left(\sigma^{2}\right)$ by the mean firing rate $(\mu)$ of every neuron from each pair; we then compared these factors in pre- and post-adaptation conditions. Fig. 4A shows the modulation of the Fano Factor for original (top) and new optimal (bottom) orientations. This factor increased from 0.49 to 0.57 for the original preferred orientation (Paired two-tailed t-test, $p<0.05$ ) and decreased from 0.58 to 0.48 for the new preferred orientation (Paired two-tailed t-test, $p<0.05$ ). These reverse trends show that spike patterns are more variable for the original optimal orientation and steadier for the new preferred orientation after adaptation. A typical example of response variability is shown in Fig. 4B, C; Gaussian tuning curves of two neurons are illustrated in Fig. 4B, the common preferred orientation for both cells is a 45 degree angle prior to adaptation. The new optimal orientation coincides with a vertically oriented angle $\left(90^{\circ}\right.$, which corresponds to the adapting orientation indicated by a black arrow in the plot) for both units post-adaptation. Probability values within each bin $(7 \mathrm{~ms})$ are plotted along the 
stimulus period of presentation $(4.1 \mathrm{~s})$ in Fig. 4C. These plots illustrate the large decorrelation for the original orientation $\left(\mathrm{R}^{2}\right.$ pre-adaptation $=0.6, \mathrm{R}^{2}$ post-adaptation $=0.09$, top), whereas the correlation is conserved (stable) for the new optimal orientation $\left(\mathrm{R}^{2}\right.$ preadaptation $=0.5, \mathrm{R}^{2}$ post-adaptation $=0.8$, bottom $)$.

\subsection{Relationship with neuronal behaviour}

Adapting neurons to a non-preferred stimulus leads to two major effects on the orientation tuning curves. Neurons acquire a new optimal orientation close to the adapter in an attractive shift. The second effect is a shift away from the adapter. Despite the fact that adaptation maintains the functional connectivity by rebalancing the excitationinhibition and maintaining this ratio, we observed significant differences between attracted and retracted conditions (Fig. 5). Indeed, for the original optimal orientation (Fig. 5A), the new optimal orientation (Fig. 5B), and the adapting orientation (Fig. 5C), the CCG-magnitude was superior for attracted neurons (paired two-tailed t-test, $p<0.05$ ). This result may indicate a predictive effect of the neuronal behaviour by estimating the strength of connectivity between neurons.

\subsection{Spike waveforms and tuning bandwidth}

Cortical processing involves a necessary interplay between different neuronal types. Two major classes of neurons are classically distinguished: regular-spiking and fast-spiking cells (Bachatene et al., 2012; Bortone et al., 2014). Fast-spiking neurons were identified by a narrow spike waveform (trough-to-peak time less than $0.5 \mathrm{~ms}$, Fig. 6A) whereas regular-spiking neurons had a longer trough-to-peak interval (greater than $0.5 \mathrm{~ms}$, Fig. 6A) (Bortone et al., 2014). An example of two neurons is shown in Fig. 6B indicating the respective spike wave-shapes of each neuron. The crosscorrelation computations were carried out between regular spiking neurons, between fast spiking neurons, and between 
both types of neurons. No significant difference was observed between cell types, that is, visual adaptation rebalances the functional connectivity strength among neurons by maintaining the global connectivity strength within neuronal networks made of regularspiking and fast-spiking neurons (Fig. 6C).

Neurons in area 17 are remarkably selective for orientation (Hubel and Wiesel, 1959). The sharpness of orientation selectivity can be gauged by measuring the bandwidth at half height of the orientation Gaussian tuning curve (Moore et al., 2005; Ringach et al., 2002). In the next analyses, we compared the peak-strength-modulation in relation to the tuning bandwidth. Fig. 6D shows the changes in the latter before and after adaptation. Cell-classes are grouped depending on whether, following adaptation, the peak-strength decreased (red dots) or increased (green dots). Note that original and new optimal orientations are used as peak markers for tuning curves. Overall, the tuning bandwidth at half magnitude (FWHM: full width at half magnitude) is slightly diminished. Interestingly, the narrowest bandwidth belongs to a particular group of neurons exhibiting the largest augmentation in the peaks-strengths (bandwidth values: green dots, original preferred orientation, non-significant from 29.41 to 21.20 , new optimal orientation significant from 26.47 to 17.33 , non-parametric Mann-Whitney test, $p<0.05$, see Fig. $6 \mathrm{D}$, right, green dots). Such a decrease strongly implies that adaptation induces the development of a jointly novel preferred orientation with a finer tuning curve. In addition, this specific group of cells exhibited the strongest enhancement of the peakstrength suggesting a closer time relationship between spike trains of involved cells.

\subsection{Dynamics of a microcircuit: restructuring the neuronal assembly by adaptation}

In previous sections, we demonstrated that the relationships between cells are modulated by adaptation. Indeed, some functional connections are strengthened while others are 
weakened, as revealed by CCG analyses. These modifications occur while both cells shift their preferred orientation to exhibit an almost identical new optimal orientation.

In the next section, we expand our analysis by constructing a peak-strength-matrix in a homogenous population, i.e., a group of simultaneously recorded neurons responding to the same original preferred stimulus and shifting their tuning curves after adaptation to the same new preferred orientation. Fig. 7 illustrates a typical example of a cluster of five cells recorded simultaneously and constituting a microcircuit with putative projections corresponding to the strength of connectivity as derived by crosscorrelation data. In Fig. 7A, orientation tuning curves (error bars indicate the standard error of the mean) and Gaussian fits of all cells are shown with their respective spike-waveforms (color codes apply to all the parts of this figure, where the black lines of spike waveforms specify the template window of spike sorting). All five cells responded similarly to the same optimal orientation ( $45^{\circ}$ angle) before adaptation. After adaptation (orientation of the adapter equals $90^{\circ}$ ), a $90^{\circ}$ oriented angle eliciting the highest discharge rate corresponds to the new acquired preferred orientation. Fig. 7B illustrates the trial-count correlation computations (TC) for all possible pairs among the cluster, the mean TC values are indicated by horizontal bars. The exceedingly weak values indicate that spike trains originate from distinct cells (see methods for details). The matrices comparing the peaksstrengths are displayed in Fig. 7C. Adaptation modified the structure of the matrices; some connections are strengthened while others are weakened, and few remain unchanged (for example, cell 1 - cell 5 pair, see Fig. 7C; cell 1 projects on cell 5 almost with equal peak-strength pre- and post-adaptation, whereas cell 5 reverse projects with varying peak-strength before and after adaptation). The values of probabilities are indicated by the color scale on the right of each matrix. These matrices allowed plotting cell-assemblies for each case and each orientation of interest. Indeed, the linkage between similarly selective neurons within a group is modified as some connections emerge, while 
others are inhibited and some cells were functionally disconnected, for instance the green cell. Fig. 7D shows a microcircuit of the presented neurons putatively connected, red lines represent the newly formed projections after adaptation (in the displayed network, the red connection indicated the change of direction from cyan cell to blue cell) and the thickness of lines highlights the strength of projections between units.

Furthermore, for the same cell-assembly, we averaged the peaks-strengths of all connections for both orientations, in order to compare the global strength of functional connectivity between the same assembly prior to and post-adaptation (Fig. 7E). The mean values for the peaks-strengths of matrices were not significant for both orientations (paired two-tailed t-test, $p=0.21$ and 0.39 for original optimal and new optimal orientations, respectively).

This analysis underscores the following: adapting cells to a non-preferred orientation induces two major effects; first there is a change of the preferred orientation and second there is a modification of the connectivity of the neuronal network and consequently its dynamics. Overall, connectivity strength is maintained and redistributed to different neurons for the broadcast of the learned feature. An example of changes of an orientation polar map is shown in Figure 7F with a new orientation-configuration established postvisual adaptation (Cattan et al., 2014).

\section{Discussion}

We examined functional time-relationships between spike trains of primary visual cortical neurons by computing crosscorrelation analyses for specific pairs of cells stimulated visually. Pairs of neurons were selected on the basis of their similar orientation-selectivity axis and post-adaptation behavior which lead to acquiring of same preferred orientation (for both neurons) after we imposed a visual "training" to non-preferred grating for 12 minutes. Crosscorrelogram data processing is a powerful in- 
situ tool for establishing the functional interactions between cells within sensory systems by indicating time-delay spiking activity between two neurons, where one neuron's spikes are set as reference to another neuron's spikes (target); therefore, it indicates putative functional projections between cells when it displays significant peaks within a short time-window reflecting direct functional relationships (Bartho et al., 2004; Denman and Contreras, 2013; Fujisawa et al., 2008; Konig et al., 1995; Perkel et al., 1967). In this report, we aimed to investigate the linkage dynamics between neuron-pairs prior and post-visual adaptation to a non-preferred oriented grating in order to reveal the extent of changes in neuronal connectivity after cells adopted new orientation-selectivity. Adaptation induced the following main results: the peaks-strengths that reflect cellular connectivity are disturbed and recalibrated after adaptation. Furthermore, the highest increase of the peak-strength belonged to cells that exhibited the sharpest bandwidth. In conclusion, there is an emergence of a novel sub-network after adaptation.

\subsection{Methodological considerations}

It was important to ask whether the disclosed functional connections were due to some physiological parameters such as anesthesia or attention. The brain state of an anaesthetized animal might control the number of responsive neurons and the magnitude of the firing rate. Rhythmic oscillations which permit the synchronization of neuronal population may also be recorded in an anesthetized state (Poulet and Petersen, 2008). Moreover, the sequence of stimuli presentations is unrelated to the spontaneous oscillatory activity of cortical rhythms of the anesthetized animals (Xing et al., 2012). Furthermore, the stimuli were applied in a random fashion with interstimulus time intervals varying from 1 to $3 \mathrm{~s}$. Since the recordings were done in the anesthetized cats, the response output of the cells for the presented stimulus cannot be attributed to the attention parameters (de Kock and Sakmann, 2009). 
Time-relationships between action potentials of neurons in the circuits may go undetected due to low firing rates of neurons; however, this does not necessarily mean that temporal locking of neurons cannot be elicited with low firing rates of neurons. It has been demonstrated that the firing rates and peaks in CCG's are unrelated (Duret et al., 2006; Rolls and Treves, 2011; Shumikhina et al., 2004).

In addition to the classical cells' isolation using spike wave-shapes, principal component analyses, and autocorrelation, it was important to ascertain that the isolated cells emerged from distinct templates; therefore we performed trial-count-correlation (TC) between every possible pair of neurons in order to compare the firing pattern between cells. To this aim, we used a $1 \mathrm{~ms}$ resolution. All correlations had a value inferior to 0.25 which ascertains that spike-trains emerged from different cells. Furthermore, we carried out these count correlations on a time-window of $4.1 \mathrm{~s}$ across all trials that corresponded to the presentation of the stimuli. Given that we divided the $4.1 \mathrm{~s}$ epoch into $1 \mathrm{~ms}$ time-bins ( $1 \mathrm{~ms}$ resolution), the probability of synchronous spikes in the same template within this short time-window is exceedingly low.

\subsection{Crosscorrelation and functional connections}

Crosscorrelation computations have been used in neurophysiological analyses in order to reveal functional linkages between spike trains of two neurons recorded simultaneously (Perkel et al., 1967). A synaptic connection can be attributed to correlation only under certain circumstances (Reid, 2012), however, a time-relationship between the firing activity two neurons may be considered as a clue to temporal projections between two cells as the temporal delay in the crosscorrelograms may suggest modifications in wiring. Temporal connections can be demonstrated by an increase in the firing probability of the postsynaptic neuron (Bharmauria et al., 2014; Reid, 2012). 
A typical CCG is generated with the aim of disclosing the significance level and strength of connectivity between two cells within a specific time-frame. Depending on the peakposition in the CCG's, the analyses revealed the spike timing of both neurons in relation to each other. However, it is worth noting that a typical CCG may lead to biased significant peaks due to the co-stimulation of both neurons at each trial of the stimulus cycle. In order to circumvent and lessen this potential bias, we employed the shuffling minus subtraction algorithm based on neuron firing rates, and subtracted it from the raw CCG's which eliminated evoked firing (Dong et al., 2008).

We examined neuronal connectivity between cell-pairs within a time-window of $\pm 5 \mathrm{~ms}$ primarily to unveil putative direct synaptic connections between cells. It has been demonstrated in the macaque MT area that neuronal crosscorrelation between pairs of cells is mostly short-termed within a time-window of 10-100 ms (Bair et al., 2001). Though in several investigations various time-windows have been taken into consideration ranging from $3 \mathrm{~ms}$ to $10 \mathrm{~ms}$ (Bartho et al., 2004) to reveal the functional connections between the involved neurons, nonetheless a time window of $\pm 5 \mathrm{~ms}$ is most frequently used and is reasonably short.

In addition to disclosing functional projections between neurons, the crosscorrelation technique allows for the evaluation of the strength of neuronal connectivity (through peak-strength) as it is significant in understanding variations of cell-assemblies in response to experience (Bock et al., 2011). We investigated the changing of probabilityindices prior to and after visual adaptation in order to uncover the modulation mechanisms of neuronal relationships (see next section for details). Indeed, this adaptation process leads to modifications of neural spiking activity attributed to push-pull mechanisms (Bachatene et al., 2012; Bachatene et al., 2013; Ghisovan et al., 2009; Kohn and Movshon, 2004). It has been demonstrated previously that long adaptation of neurons 
to a non-preferred stimulus, which is orientation in our experiments, for the most part leads to a decrease in firing activity for the original optimal orientation, and in contrast, a firing increase for the new preferred orientation (Bachatene et al., 2012; Bachatene et al., 2013; Ghisovan et al., 2009). It was relevant to explore whether the probability modulation of neuronal connectivity is related to the firing rates of neurons, as previously shown. However, we found no significant correlation between the peak-strengths and the firing rates in our results, as previously described (Duret et al., 2006; Ghisovan et al., 2008; Rolls and Treves, 2011). In addition, results indicated no relationship between spike waveforms which are attributed to specific putative cellular types, and peak strength fluctuations post-adaptation.

\subsection{Neuronal connectivity and orientation-selectivity}

Neurons within the primary visual cortex are "orientation-detectors," and respond maximally to a properly oriented stimulus presented in their receptive field (Hubel and Wiesel, 1959). Several studies have demonstrated the ability of V1 neurons to adjust their stimulus-selectivity in order to restructure the primary visual cortex as an "adapted cortex" to the imposed visual environment by a persistent adaptation to a specific stimulus. For instance, such modifications have been reported for orientation (Bachatene et al., 2012; Bachatene et al., 2013; Dragoi et al., 2000; Ghisovan et al., 2009; Kohn and Movshon, 2004), spatial frequency (Marshansky et al., 2011), direction of motion (Kohn and Movshon, 2004) and speed (Movshon, 1975). By changing their preferred features, neurons acquire new optimal properties for a short or long period of time depending on the visual training duration (Bachatene et al., 2013; Ghisovan et al., 2009; Patterson et al., 2013). In V1, cells are scattered within cortical domains and connected to each other

laterally and vertically.

Horizontal connections are characterized as long range connections between neuron 
s preferring similar stimulus features (Das and Gilbert, 1995; Sompolinsky et al., 1990) which are functionally connected to each other at large distances (Hata et al., 1991; Ts'o et al., 1986). Vertical connections are described as inputs to layers II and III from layer IV of the visual cortex (Stratford et al., 1996; Yoshimura et al., 2000) which receives its inputs from LGN (Alonso et al., 1996). From layers II and III, the connections descend to layers V and VI (Stratford et al., 1996).

As suggested by crosscorrelogram analysis, neuronal connectivity has been reported to be high between cells with similar preferred parameters due in part to specific connections between cortical domains having similar tuning properties (Alloway and Roy, 2002; Alonso et al., 1996; Bartho et al., 2004; Csicsvari et al., 1998; Yoshimura et al., 2005). Indeed the correlation between neurons sharing reciprocal tuning properties is well established (Kohn and Smith, 2005; Zohary et al., 1994). Neuronal connections within the cortex are very specific, mostly depending on the properties of the responses of neurons (Alonso, 2002). For instance, in macaque visual cortex, it has been shown that correlation varies with stimulus condition and this variation is stronger between neurons sharing similar direction tuning curves (Bair et al., 2001). In mouse primary visual cortex, it has been reported that significant pair-wise synchrony is related to orientation preference between neurons (Denman and Contreras, 2013). Neuronal connectivity can also be related to specific stimulus preference; it has been demonstrated that in mouse visual cortex, the similarity of neuronal activity to a specific feature of the stimulus affects the connection-probabilities; for example, neurons sharing similar selectivity for oriented stimuli exhibit more connectedness with each other than neurons having orthogonal orientation selectivity (Ko et al., 2011).

These findings provide evidence that stimuli features influence the temporal pattern of neuronal activity (Gray et al., 1990). Nevertheless, following plastic modifications, connectivity patterns among neuronal pairs in V1 have yet to be explored. In the present 
paper, we sought to examine how the peaks-strengths calculated from crosscorrelation analyses between pair-wise neurons sharing stimulus-feature selectivity are modulated following a long duration visual "training."

Following the critical period, neurons programmed to connect to distinctive neurons that share homogenous orientation-selectivity start responding to a new acquired orientation after visual adaptation, and therefore switch on to connect a different neuronal population to participate in a different sub-network of cells. Indeed, some authors have demonstrated in mouse visual cortex a reorganization of the connectivity configuration. This is related to a higher proportion of links between neurons with similar visual responses, while connections were eliminated between visually unresponsive units, yet the overall connectedness remained unchanged (Ko et al., 2013).

Although peaks-strengths on average were not significantly different, individual pairs appeared to increase or decrease their respective peaks, suggesting that the adaptation process redeploys the strength of projections between neurons to restructure the entire linkage-dynamic of the neuronal population in response to the changing stimuli environment. For instance, the forceful presentation of a particular stimulus (a different orientation in our experiments) results in the disappearance of some relationships and the appearance of new projections. Understanding the temporal relations between neurons within sub-networks could help us to decipher how visual processing is altered under specific changes of environmental conditions and how the plastic capacity of neuronal networks lead to the acquisition of new features. Although our stimulation conditions are artificial (stimulus exposure), it still mimics training or forcing cells to respond to a particular trigger feature which in turn, may serve as basis for learning process.

An alternative possibility may be hypothesized. Neurons are connected with excitatory (E) and inhibitory (I) fibers and their spiking output is the net outcome of these antagonistic influences. Thus adaptation may strengthen and weaken excitatory and 
inhibitory connections respectively in order to unlock cellular time-relationships in involved cortical areas whereby a new E-I equilibrium might be considered as a possible scenario for the neuronal variability dynamic after visual adaptation. The fluctuations of neuronal alterability due to adaptation effects may rebalance the E-I ratio and contribute to the stability of the total inter-neuronal variability (Kasamatsu et al., 2001). It has been shown in layers $2 / 3$ of the mouse visual cortex that E-I ratios are equalized across pyramidal cells (Xue et al., 2014). Our results are in concordance with the equalization of excitation-inhibition ratios after adaptation (Fig. 7D, E).

This is in line with recent findings which assign an important contribution of adaptation to neuronal homeostasis within a population of cells sharing neuronal response uniformity, as well as visual feature selectivity (Benucci et al., 2013). We reported that adaptation restructures the neural code following plasticity by maintaining the connectivity strength within the cellular network and redistributing this linkage to different neurons within the assembly (see Fig. 7D, E). In models of cross-orientation, feature-selectivity is induced by inhibitory connections between cells with different orientation preferences (Alonso, 2002). Furthermore, it has been shown that in relation to differences in orientation-selectivity between two neurons, excitatory, inhibitory and common input correlations occur mostly in neuronal pairs with a difference of orientation preference inferior to 45 degrees (Hata et al., 1991). Moreover, adaptation may provide a 'window of opportunity' for recipient neurons to modify excitatory/inhibitory balance in order to reorganize the spiking cortical activity (Stanley, 2013). Indeed, complex feedforward-feedback mechanisms emerge within the primary visual cortex in order to reorganize the signal processing following plasticity (Fahle, 2004).

Therefore, we postulate that orientation-selective neuronal pairs modify the strength of connections following adaptation in order to recalibrate the neural code. 
Such rapid changes may not be accounted by structural modifications, there are most likely due to modification of equilibrium between excitation and inhibition influences to a recorded cell which allows silent synapses to become effective. For instance, in mouse visual cortex, it has been demonstrated that a single dendritic branch receive synaptic drives from a large spectrum of orientations (Jia et al., 2010).

Excitatory and inhibitory cells coordinate time-related firing activity to maintain the neuronal population output during visual information processing.

\section{Conclusion}

Visual adaptation of neurons to non-optimal stimuli suggests the adaptability of neuronal code to visual stimuli. In the primary visual cortex, neurons respond in coordination with neighboring cells to the trigger features, and encode stimuli properties by constituting cell assemblies (Kampa et al., 2011; Lee and Reid, 2011; Wallace and Kerr, 2010) wherein cells are anatomically connected by forming synaptic contacts or by interacting through synchronous time-relationships. To reveal how this local circuitry of different neurons in the visual cortex is set up and modulated in response to different visual stimulation conditions is of prime importance to understanding the mechanisms of information processing.

\section{Experimental procedures}

\subsection{Ethical approval}

Animal surgery procedures and electrophysiological recordings followed the guidelines of the Canadian Council on Animal Care and were approved by the Institutional Animal Care and Use Committee of the University of Montreal. Animals were supplied by the Division of Animal Resources of the University of Montreal. The experiments were 
conducted in accordance with the Guide for Care and Use of Laboratory Animals of the National Institutes of Health (USA).

\subsection{Animal surgery}

Electrophysiological recordings were performed using multielectrodes within V1 area of adult domestic cats (Felis catus). Eleven adult cats $(2.5-3.5 \mathrm{~kg}$, age 12-24 months) of either sex, sedated with acepromazine maleate (Atravet, Wyeth-Ayerst, Guelph, ON, Canada; $1 \mathrm{mg} \mathrm{kg}^{-1}$, intramuscular) and atropine sulfate (ATRO-SA, Rafter, Calgary, AB, Canada; $0.04 \mathrm{mg} \mathrm{kg}^{-1}$, intramuscular), were anaesthetized with ketamine hydrochloride (Rogarsetic, Pfizer, Kirkland, QC, Canada; $25 \mathrm{mg} \mathrm{kg}^{-1}$, intramuscular) maintained with 0.3\% isoflurane (AErrane, Baxter, Toronto, ON, Canada). Lidocaine hydrochloride (Xylocaine, AstraZeneca, Missis-sauga, ON, Canada; 2\%) was injected subcutaneously as a local anaesthetic during surgery. A tracheotomy was performed for artificial ventilation, and one forelimb vein was cannulated. Animals were then placed in a stereotaxic apparatus. Xylocaine gel (Astra Pharma, Mississauga, ON, Canada; 5\%) was applied on the pressure points. For the remaining preparations and recording, paralysis was induced with $40 \mathrm{mg}$ and maintained with $10 \mathrm{mg} \mathrm{kg}^{-1} \mathrm{~h}^{-1}$ gallamine triethiodide (Flaxedil, Sigma Chemical, St. Louis, MO, USA; intravenous) administered in 5\% dextrose lactated Ringer's nutritive solution. General anaesthesia was maintained by artificial ventilation with a mixture of $\mathrm{N}_{2} \mathrm{O} / \mathrm{O}_{2}$ (70:30) supplemented with $0.5 \%$ isoflurane (AErrane, Baxter, Toronto, ON, Canada) for the duration of the experiment. Proper depth of anaesthesia was ensured throughout the experiment by monitoring the EEG, the electrocardiogram and expired $\mathrm{CO}_{2}$. In addition the heart rate remained unmodified after skin stimulation. The end-tidal $\mathrm{CO}_{2}$ partial pressure was kept constant between 25 and $30 \mathrm{mmHg}$. A heated pad was used to maintain a body temperature of $37.5^{\circ} \mathrm{C}$. Tribrissen (Schering-Plough, Pointe-Claire, QC, Canada; $30 \mathrm{mg} \mathrm{kg}^{-1}$ per day, 


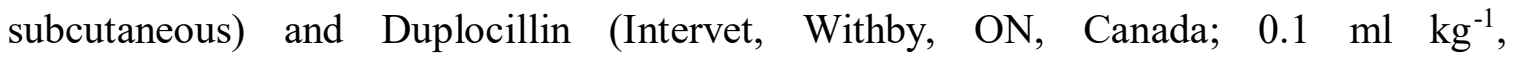
intramuscular) were administered to the animals to prevent bacterial infection. The pupils were dilated with atropine sulfate (Isopto-Atropine, Alcon, Mississauga, ON, Canada; $1 \%)$ and the nictitating membranes were retracted with phenylephrine hydrochloride (Mydfrin, Alcon, Mississauga, ON, Canada; 2.5\%). The loci of the area centrales were inferred from the positions of the blind spots, which were ophthalmoscopically focused and back projected onto a translucent screen. In order to verify the stability of the eye this procedure was repeated at the end of tests. Plano contact lenses with artificial pupils (5 mm diameter) were placed on the cat's eyes to prevent the cornea from drying (University of Montréal, PQ, Canada).

At the end of each experiment, euthanasia was achieved with a lethal dose of pentobarbital sodium (Somnotol, MTC Pharmaceuticals, Cambridge, ON, Canada; 100 $\mathrm{mg} \mathrm{kg}^{-1}$ ) by intravenous injection.

\subsection{Electrophysiology}

Multi-unit activity in the primary visual cortex was carried out with tungsten microelectrode (Frederick Haer \& Co, Bowdoinham, ME, USA; 2-10 M 2 at $1 \mathrm{kHz}$ ). Each set of electrodes, consisting of a four microelectrode in linear array (inter-electrode spacing of $400 \mu$ ) enclosed in stainless steel tubing, was controlled by a separate micromanipulator. The signal from the microelectrodes was amplified, band-pass filtered $(300 \mathrm{~Hz}-3 \mathrm{kHz}$ ), digitized and recorded with a $0.05 \mathrm{~ms}$ temporal resolution (Spike2, CED, Cambridge, England). We recorded at an average of 400-500 $\mu \mathrm{m}$ cortical depth from two recording sites (Average of three neurons recorded per electrode per session). Action potentials were sorted out using a window discriminator for further off-line analyses. Multiunit signals from one electrode included well-isolated single units. The spike sorting method was based on cluster classification in reduced space (Spike2, CED). 
The stability of each cell's activity across conditions was verified qualitatively by visual control of the clusters disposition and of the waveforms shape. Auto-correlograms and principal component analysis were systematically performed to insure proper single cell capture (refractory period for auto-correlograms). Spike-count correlation was used to ascertain that spikes emerged from different neurons. $85 \%$ of neurons were included in the data analyses.

\subsection{Visual stimulation}

Stimulation was monocular (dominant eye, the opposite eye was shut). After clearly detectable activity was obtained, the multiunit receptive fields (RF) were mapped as the minimum response fields (Barlow et al., 1967) by using a hand-held ophthalmoscope. RF edges were determined by moving a light bar from the periphery towards the center until a response was elicited. Eye-screen distance was $57 \mathrm{~cm}$. These preliminary tests revealed qualitative properties such as dimensions, velocity preference, orientation and directional selectivity. Visual stimuli were generated with a VSG 2/5 graphic board (Cambridge Research Systems, Rochester, England) and displayed on a 21-in. monitor (Sony GDMF520 Trinitron, Tokyo, Japan) placed $57 \mathrm{~cm}$ from the cat's eyes, with $1024 \times 768$ pixels, running at $100 \mathrm{~Hz}$ frame refresh. Stimuli were drifting sine-wave grating patches $\left(\sim 2^{\circ}\right.$ to $5^{\circ}$ ) covering the excitatory RF (Maffei and Fiorentini, 1973). Patches characteristics were set to evoke optimal responses: contrast at $80 \%$, mean luminance at $40 \mathrm{Cd} \cdot \mathrm{m}^{2}$, optimal spatial and temporal frequencies set within the $0.1-0.5$ cycles/deg. and $1.0-2.0 \mathrm{~Hz}$ range

respectively. The blank screen was uniformly gray $\left(\sim 35 \mathrm{~cd} \mathrm{~m}^{-2}\right)$. In all cases the above parameters were chosen with the aim of evoking the maximal discharges. V1 neurons are known to respond well to sine wave drifting gratings (Bardy et al., 2006). After manual RF characterization, nine oriented stimuli centered on the preferred orientation were selected and used for the rest of the experiment. With a $22.5^{\circ}$ interval between 
orientations, tuning curves covered $180^{\circ}$. Test orientations were applied in random order. Each oriented stimulus was presented in blocks of 25 trials lasting $4.1 \mathrm{~s}$ each, with a random inter-trial interval (1.0-3.0 s) during which no stimuli were presented. Thus, a recording session lasted for 25-30 min. Peri-stimulus time histograms were recorded. Once control orientation tuning curves were characterized, an adapting non-preferred stimulus was presented continuously for $12 \mathrm{~min}$. The adapting stimulus was a drifting grating whose orientation was randomly selected in the range 22.5 to $67.5^{\circ}$ off of the neuron's preferred orientation (Fig. 8A). All other stimulus parameters were kept constant, at control values, throughout the recordings. During this adaptation period no recordings were performed. Immediately after adaptation, orientation tuning curves was measured starting with the adapting and control preferred orientations to prevent an unlikely premature recovery, as these two orientations were the center of interest, while the remaining orientations were recorded in random order.

\subsection{Cells' isolation}

Given that the multi-unit activity was recorded concurrently from same tips using multielectrodes, it was essential to ascertain that cells were well isolated, because the same unit may exhibit sufficiently different waveforms (for instance magnitude in relation to distance between recording tip), and thus belong to different clusters in principal component analysis. Cell-separation was based on spike-waveforms, cluster-isolation using first principal components analyses, autocorrelograms and trial-count correlation (TC).

Cluster analysis was performed using Spike2 (CED, Cambridge, England) in a 3dimensional plot. The isolation distance was calculated using the Mahalanobis distance; it defines boundaries of constant probability around the multi-dimensional center of the distribution. This estimation allows the separation of a cluster from the 
nearest cluster. All clusters within Mahalanobis distance of 2.5 were considered for analysis (Bharmauria et al., 2014).

Trial-count correlation (TC) denotes the trial by trial Pearson correlation-coefficient between simultaneously recorded firing of two neurons in response to the presentation of the identical stimulus. In response to the same grating, the same unit fires identically regardless of spike amplitude. In order to eliminate such occurrences, we correlated neural activities of each cell-pair for every applied trial (25 trials, same stimulus, duration $4.1 \mathrm{~s})$. Given that optimal orientations eliciting maximal firing rates were chosen for this computation as well as a relatively long time-window analysis (4.1 s), we would have expected a high value for correlation if it had been the same unit, because such a timewindow analysis is sufficiently large to capture the full strength of correlation (Bair et al., 2001; Kohn and Smith, 2005; Reich et al., 2001). Indeed correlations would be underestimated if the counting window is too short (Cohen and Kohn, 2011). In spite of such conditions, the found correlations were exceedingly low (average $\mathrm{TC}=-0.005$, see Fig. 8B). Pairs exhibiting correlations superior to 0.25 were eliminated from further analyses (as a general rule). This very weak correlation in spike response patterns (close to zero) indicates that the spikes arise from different units, hence the cells fire in an independent fashion. Fig. 8B illustrates the trial-count correlation analysis performed for the cells and recorded from the same electrode tip within each group.

Fig. 8B depicts the TC values for all trials $(\mathrm{n}=1894)$ accumulated over all selected neuron-pairs (the average TC value for each cell pair equals to -0.005). The distribution of the average TC for each neuronal pair is shown in the figure (gray curve represents the normal distribution curve). The correlation values are clustered around zero and never exceed 0.25, which is in agreement with earlier published data (Cohen and Kohn, 2011; Ecker et al., 2010; Maruyama and Ito, 2013). This ascertains that firing patterns of the two selected neurons are different and ensures the cells' isolation. 
Autocorrelograms preclude contamination by spikes of other units. Anesthesia and animal paralysis reduce the possibility of similar modulations of firing patterns in cells of same clusters due to rapid eye motion or attention (de Kock and Sakmann, 2009).

\subsection{Data analysis and statistical computations}

Once single cells were sorted out off-line from multi-unit spike trains accumulated during data acquisition, orientation tuning curves were constructed from raw data. Because orientation tuning is best described by Gaussian-like functions, we fitted our raw data with the von Mises function (Swindale, 1998). This allowed us to determine with precision the preferred orientation of neurons and then measure shifts in orientation preference. The von Mises function is defined as:

$M(\theta)=A \times e^{b[\cos (\theta-c)]}+d$

where $\mathrm{A}$ is the value of the function at the preferred orientation, $\mathrm{c}$, and $\mathrm{b}$ is a width parameter. An additional parameter, d, represents the spontaneous firing rate of the cell (Kohn and Movshon, 2004; Swindale, 1998). The above calculations are necessary because tuning curves derived from raw data may be imperfect in determining the preferred orientation since the interval between the stimulus orientations is relatively large, $22.5^{\circ}$. In the cat, over $90 \%$ of $\mathrm{V} 1$ neurons are well tuned to stimulus orientation (Bishop and Henry, 1972). It was, however, necessary to ensure that cells in our sample were properly tuned for orientation. We measured an orientation selectivity index (OSI) by dividing the firing rate at orthogonal orientations by the firing rate for the preferred orientation, and subtracting the result from one (Liao et al., 2004; Ramoa et al., 2001). 
The closer the OSI is to one, the stronger the orientation selectivity. Adaptation induced shifts were measured as the distance between peak positions of the fitted tuning curves before and after conditioning. To assess the statistical significance of tuning shifts, curve fits were generated separately for each of the 25 trials, and the mean difference was tested by a paired $t$-test. Shifts in preferred orientation larger than $5^{\circ}$ were statistically significant (paired sample two-tailed $t$-test, $p<0.05$ ). Tuning bandwidths were calculated based on the full width at half magnitude of the Gaussian tuning curves. Statistical analyses were performed with Graphpad Prism (GraphPad Software Inc., La Jolla, California, USA). Student's t-tests were computed to compare the peaks-strengths between control and post-adaptation conditions, and to compare bandwidths between different classes. Ninety-five percent confidence-interval was used. Gaussian curves were fitted using the following equation:

$y=y 0+\left(A \div\left(w \times \sqrt{\left(\frac{\pi}{2}\right)}\right)\right) \times e^{\left(-2 \times\left(\frac{(x-x c)}{w}\right)^{2}\right)}$

where $\mathrm{y} 0$ is the offset, $\mathrm{xc}$ is the center, $\mathrm{w}$ is the width and A represents the area.

Non-linear regression analyses were computed using this equation:

$$
y=y 0+A 1 \times\left(1-e^{\left(\frac{-x}{t 1}\right)}\right)+A 2 \times\left(1-e^{\left(\frac{-x}{t 2}\right)}\right)
$$

where $\mathrm{y} 0$ is the offset, $\mathrm{A} 1$ and $\mathrm{A} 2$ are the amplitudes, $\mathrm{t} 1$ and $\mathrm{t} 2$ are the widths.

\subsection{Crosscorrelograms, shift predictor and confidence limits}


We generated CCG's between simultaneously recorded spike trains of cell-pairs at specific orientations, i.e. the original optimal orientation and the new optimal orientation of each pair of neurons. Cell-pairs were selected based on their similarity of spiking discharge for these two preferred orientations using an orientation range of \pm 22.5 degrees.

CCG's were performed in order to compare the spike distribution of each neuron of the pair within a time-frame; one neuron is set as reference and the second as target; this allows us to show the firing of the target neuron at a specific time-spread in relation to the firing of the reference neuron.

Time axis is divided into bins. The first bin is defined as: XMin, XMin+Bin. The next bin is $\mathrm{XMin}+\mathrm{Bin}, \mathrm{XMin}+\mathrm{Bin} * 2$, etc. We calculated the distances from each spike to all spikes of the spike train as follows:

$d[i]=t s[i]-\operatorname{ref}[k]$

where ts[i] represents the spike train, and ref[k] is each timestamp.

Bin counts were then divided by the number of reference events to normalize the counts per bin into probabilities. Fig. 8D illustrates the parameters of CCG's; the red line represents the $95 \%$ statistical threshold for the significance of the bins. Each bin-width was set at $1 \mathrm{~ms}, \mathrm{Y}$-axis corresponds to the peak-strength calculated from the counts/bin as follows:

$P=F \times b$

where $\mathrm{F}$ is the neuron frequency and $\mathrm{b}$ represents the bin size of the calculated firing of the neuron (Abeles, 1982). 
The neuron frequency $\mathrm{F}$ was calculated as follows:

$$
F=\frac{N}{T}
$$

where $\mathrm{T}$ represents the total time interval and $\mathrm{N}$ the number of spikes within this interval. The $95 \%$ confidence limit was calculated assuming that the expected bin count (C) has a Poisson distribution:

$C=P \times N r e f$

where Nref is the number of reference events.

The $95 \%$ confidence limit is calculated as follows:

Low Conf.$=x$ such that Prob $(S<x)=0.005$

High Conf.$=y$ such that Prob $(S>y)=0.005$

where S represents a random variable which has a Poisson distribution with parameter C.

The bins which fit into the $\pm 1 \mathrm{~ms}$ around the center were classified as synchrony events (putative common afferent input to both neurons). Raw CCG's were corrected by subtracting a shift-predictor algorithm in order to eliminate the putative significant peaks due to the simultaneous stimulation of both cells during each trial. We used linear regression analysis with $95 \%$ confidence interval for the entire population of the recorded 
neuron-pairs to determine the modulation of the peak-strength for the two major specific

preferred stimuli (original and newly acquired orientations).

\section{Acknowledgments}

This work was supported by grants to S.M.

The Natural Sciences and Engineering Research Council of Canada.

Fonds québécois de la recherche sur la nature et les technologies.

\section{References}

Abeles, M., 1982. Quantification, smoothing, and confidence limits for single-units' histograms. J Neurosci Methods. 5, 317-25.

Alloway, K.D., Roy, S.A., 2002. Conditional cross-correlation analysis of thalamocortical neurotransmission. Behav Brain Res. 135, 191-6.

Alonso, J.M., Usrey, W.M., Reid, R.C., 1996. Precisely correlated firing in cells of the lateral geniculate nucleus. Nature. 383, 815-9.

Alonso, J.M., 2002. Neural connections and receptive field properties in the primary visual cortex. Neuroscientist. 8, 443-56.

Bachatene, L., Bharmauria, V., Rouat, J., Molotchnikoff, S., 2012. Adaptation-induced plasticity and spike waveforms in cat visual cortex. Neuroreport. 23, 88-92.

Bachatene, L., Bharmauria, V., Cattan, S., Molotchnikoff, S., 2013. Fluoxetine and serotonin facilitate attractive-adaptation-induced orientation plasticity in adult cat visual cortex. Eur J Neurosci. 38, 2065-77.

Bair, W., Zohary, E., Newsome, W.T., 2001. Correlated firing in macaque visual area MT: time scales and relationship to behavior. J Neurosci. 21, 1676-97.

Bardy, C., Huang, J.Y., Wang, C., FitzGibbon, T., Dreher, B., 2006. 'Simplification' of responses of complex cells in cat striate cortex: suppressive surrounds and 'feedback' inactivation. J Physiol. 574, 731-50.

Barlow, H.B., Blakemore, C., Pettigrew, J.D., 1967. The neural mechanism of binocular depth discrimination. J Physiol. 193, 327-42.

Bartho, P., Hirase, H., Monconduit, L., Zugaro, M., Harris, K.D., Buzsaki, G., 2004. Characterization of neocortical principal cells and interneurons by network interactions and extracellular features. J Neurophysiol. 92, 600-8.

Benucci, A., Saleem, A.B., Carandini, M., 2013. Adaptation maintains population homeostasis in primary visual cortex. Nat Neurosci. 16, 724-9.

Bharmauria, V., Bachatene, L., Cattan, S., Rouat, J., Molotchnikoff, S., 2014. Synergistic activity between primary visual neurons. Neuroscience. 268, 255-64. 
Bishop, P.O., Henry, G.H., 1972. Striate neurons: receptive field concepts. Invest Ophthalmol. 11, 346-54.

Bock, D.D., Lee, W.C., Kerlin, A.M., Andermann, M.L., Hood, G., Wetzel, A.W., Yurgenson, S., Soucy, E.R., Kim, H.S., Reid, R.C., 2011. Network anatomy and in vivo physiology of visual cortical neurons. Nature. 471, 177-82.

Bortone, D.S., Olsen, S.R., Scanziani, M., 2014. Translaminar inhibitory cells recruited by layer 6 corticothalamic neurons suppress visual cortex. Neuron. 82, 47485.

Cattan, S., Bachatene, L., Bharmauria, V., Jeyabalaratnam, J., Milleret, C., Molotchnikoff, S., 2014. Comparative analysis of orientation maps in areas 17 and 18 of the cat primary visual cortex following adaptation. Eur J Neurosci. 40, 2554-63.

Cohen, M.R., Kohn, A., 2011. Measuring and interpreting neuronal correlations. Nat Neurosci. 14, 811-9.

Csicsvari, J., Hirase, H., Czurko, A., Buzsaki, G., 1998. Reliability and state dependence of pyramidal cell-interneuron synapses in the hippocampus: an ensemble approach in the behaving rat. Neuron. 21, 179-89.

Das, A., Gilbert, C.D., 1995. Long-range horizontal connections and their role in cortical reorganization revealed by optical recording of cat primary visual cortex. Nature. 375, 780-4.

de Kock, C.P., Sakmann, B., 2009. Spiking in primary somatosensory cortex during natural whisking in awake head-restrained rats is cell-type specific. Proc Natl Acad Sci U S A. 106, 16446-50.

Denman, D.J., Contreras, D., 2013. The Structure of Pairwise Correlation in Mouse Primary Visual Cortex Reveals Functional Organization in the Absence of an Orientation Map. Cereb Cortex.

Dong, Y., Mihalas, S., Qiu, F., von der Heydt, R., Niebur, E., 2008. Synchrony and the binding problem in macaque visual cortex. J Vis. 8, 30 1-16.

Dragoi, V., Sharma, J., Sur, M., 2000. Adaptation-induced plasticity of orientation tuning in adult visual cortex. Neuron. 28, 287-98.

Duret, F., Shumikhina, S., Molotchnikoff, S., 2006. Neuron participation in a synchrony-encoding assembly. BMC Neurosci. 7, 72.

Ecker, A.S., Berens, P., Keliris, G.A., Bethge, M., Logothetis, N.K., Tolias, A.S., 2010. Decorrelated neuronal firing in cortical microcircuits. Science. 327, 584-7.

Fahle, M., 2004. Perceptual learning: a case for early selection. J Vis. 4, 879-90.

Fujisawa, S., Amarasingham, A., Harrison, M.T., Buzsaki, G., 2008. Behaviordependent short-term assembly dynamics in the medial prefrontal cortex. Nat Neurosci. 11, 823-33.

Ghisovan, N., Nemri, A., Shumikhina, S., Molotchnikoff, S., 2008. Synchrony between orientation-selective neurons is modulated during adaptation-induced plasticity in cat visual cortex. BMC Neurosci. 9, 60.

Ghisovan, N., Nemri, A., Shumikhina, S., Molotchnikoff, S., 2009. Long adaptation reveals mostly attractive shifts of orientation tuning in cat primary visual cortex. Neuroscience. 164, 1274-83.

Godde, B., Leonhardt, R., Cords, S.M., Dinse, H.R., 2002. Plasticity of orientation preference maps in the visual cortex of adult cats. Proc Natl Acad Sci U S A. 99, 6352-7.

Gray, C.M., Engel, A.K., Konig, P., Singer, W., 1990. Stimulus-Dependent Neuronal Oscillations in Cat Visual Cortex: Receptive Field Properties and Feature Dependence. Eur J Neurosci. 2, 607-619. 
Hata, Y., Tsumoto, T., Sato, H., Tamura, H., 1991. Horizontal interactions between visual cortical neurones studied by cross-correlation analysis in the cat. J Physiol. 441, 593-614.

Hubel, D.H., Wiesel, T.N., 1959. Receptive fields of single neurones in the cat's striate cortex. J Physiol. 148, 574-91.

Hubel, D.H., Wiesel, T.N., 1968. Receptive fields and functional architecture of monkey striate cortex. J Physiol. 195, 215-43.

Jia, H., Rochefort, N.L., Chen, X., Konnerth, A., 2010. Dendritic organization of sensory input to cortical neurons in vivo. Nature. 464, 1307-12.

Kampa, B.M., Roth, M.M., Gobel, W., Helmchen, F., 2011. Representation of visual scenes by local neuronal populations in layer $2 / 3$ of mouse visual cortex. Front Neural Circuits. 5, 18.

Kara, P., Reid, R.C., 2003. Efficacy of retinal spikes in driving cortical responses. J Neurosci. 23, 8547-57.

Kasamatsu, T., Polat, U., Pettet, M.W., Norcia, A.M., 2001. Colinear facilitation promotes reliability of single-cell responses in cat striate cortex. Exp Brain Res. 138, 163-72.

Ko, H., Hofer, S.B., Pichler, B., Buchanan, K.A., Sjostrom, P.J., Mrsic-Flogel, T.D., 2011. Functional specificity of local synaptic connections in neocortical networks. Nature. 473, 87-91.

Ko, H., Cossell, L., Baragli, C., Antolik, J., Clopath, C., Hofer, S.B., Mrsic-Flogel, T.D., 2013. The emergence of functional microcircuits in visual cortex. Nature. 496, 96-100.

Kohn, A., Movshon, J.A., 2004. Adaptation changes the direction tuning of macaque MT neurons. Nat Neurosci. 7, 764-72.

Kohn, A., Smith, M.A., 2005. Stimulus dependence of neuronal correlation in primary visual cortex of the macaque. J Neurosci. 25, 3661-73.

Konig, P., Engel, A.K., Roelfsema, P.R., Singer, W., 1995. How precise is neuronal synchronization? Neural Comput. 7, 469-85.

Lee, W.C., Reid, R.C., 2011. Specificity and randomness: structure-function relationships in neural circuits. Curr Opin Neurobiol. 21, 801-7.

Liao, D.S., Krahe, T.E., Prusky, G.T., Medina, A.E., Ramoa, A.S., 2004. Recovery of cortical binocularity and orientation selectivity after the critical period for ocular dominance plasticity. J Neurophysiol. 92, 2113-21.

Maffei, L., Fiorentini, A., 1973. The visual cortex as a spatial frequency analyser. Vision Res. 13, 1255-67.

Marshansky, S., Shumikhina, S., Molotchnikoff, S., 2011. Repetitive adaptation induces plasticity of spatial frequency tuning in cat primary visual cortex. Neuroscience. 172, 355-65.

Maruyama, Y., Ito, H., 2013. Diversity, heterogeneity and orientation-dependent variation of spike count correlation in the cat visual cortex. Eur J Neurosci. 38, 3611-27.

Moore, B.D.t., Alitto, H.J., Usrey, W.M., 2005. Orientation tuning, but not direction selectivity, is invariant to temporal frequency in primary visual cortex. J Neurophysiol. 94, 1336-45.

Movshon, J.A., 1975. The velocity tuning of single units in cat striate cortex. J Physiol. 249, 445-68.

Patterson, C.A., Wissig, S.C., Kohn, A., 2013. Distinct effects of brief and prolonged adaptation on orientation tuning in primary visual cortex. J Neurosci. 33, $532-43$. 
Perkel, D.H., Gerstein, G.L., Moore, G.P., 1967. Neuronal spike trains and stochastic point processes. I. The single spike train. Biophys J. 7, 391-418.

Poulet, J.F., Petersen, C.C., 2008. Internal brain state regulates membrane potential synchrony in barrel cortex of behaving mice. Nature. 454, 881-5.

Ramoa, A.S., Mower, A.F., Liao, D., Jafri, S.I., 2001. Suppression of cortical NMDA receptor function prevents development of orientation selectivity in the primary visual cortex. J Neurosci. 21, 4299-309.

Reich, D.S., Mechler, F., Victor, J.D., 2001. Independent and redundant information in nearby cortical neurons. Science. 294, 2566-8.

Reid, R.C., 2012. From functional architecture to functional connectomics. Neuron. 75, 209-17.

Ringach, D.L., Shapley, R.M., Hawken, M.J., 2002. Orientation selectivity in macaque V1: diversity and laminar dependence. J Neurosci. 22, 5639-51.

Rolls, E.T., Treves, A., 2011. The neuronal encoding of information in the brain. Prog Neurobiol. 95, 448-90.

Shumikhina, S., Guay, J., Duret, F., Molotchnikoff, S., 2004. Contextual modulation of synchronization to random dots in the cat visual cortex. Exp Brain Res. 158, 223-32.

Sompolinsky, H., Golomb, D., Kleinfeld, D., 1990. Global processing of visual stimuli in a neural network of coupled oscillators. Proc Natl Acad Sci U S A. 87, 72004.

Stanley, G.B., 2013. Reading and writing the neural code. Nat Neurosci. 16, 259-63.

Stepanyants, A., Hirsch, J.A., Martinez, L.M., Kisvarday, Z.F., Ferecsko, A.S., Chklovskii, D.B., 2008. Local potential connectivity in cat primary visual cortex. Cereb Cortex. 18, 13-28.

Stratford, K.J., Tarczy-Hornoch, K., Martin, K.A., Bannister, N.J., Jack, J.J., 1996. Excitatory synaptic inputs to spiny stellate cells in cat visual cortex. Nature. 382, 258-61.

Swindale, N.V., 1998. Orientation tuning curves: empirical description and estimation of parameters. Biol Cybern. 78, 45-56.

Ts'o, D.Y., Gilbert, C.D., Wiesel, T.N., 1986. Relationships between horizontal interactions and functional architecture in cat striate cortex as revealed by cross-correlation analysis. J Neurosci. 6, 1160-70.

Wallace, D.J., Kerr, J.N., 2010. Chasing the cell assembly. Curr Opin Neurobiol.

Wise, A.K., Cerminara, N.L., Marple-Horvat, D.E., Apps, R., 2010. Mechanisms of synchronous activity in cerebellar Purkinje cells. J Physiol. 588, 2373-90.

Xing, D., Shen, Y., Burns, S., Yeh, C.I., Shapley, R., Li, W., 2012. Stochastic generation of gamma-band activity in primary visual cortex of awake and anesthetized monkeys. J Neurosci. 32, 13873-80a.

Xue, M., Atallah, B.V., Scanziani, M., 2014. Equalizing excitation-inhibition ratios across visual cortical neurons. Nature. 511, 596-600.

Yoshimura, Y., Sato, H., Imamura, K., Watanabe, Y., 2000. Properties of horizontal and vertical inputs to pyramidal cells in the superficial layers of the cat visual cortex. J Neurosci. 20, 1931-40.

Yoshimura, Y., Dantzker, J.L., Callaway, E.M., 2005. Excitatory cortical neurons form fine-scale functional networks. Nature. 433, 868-73.

Zohary, E., Shadlen, M.N., Newsome, W.T., 1994. Correlated neuronal discharge rate and its implications for psychophysical performance. Nature. 370, 140-3. 


\section{Legends of figures}

Fig. 1 Typical example. (A) Two neurons (pink and blue units) sorted using spike waveforms, principal component analyses, and autocorrelograms.

(B) Raster plots and peri-stimulus time histograms for both cells (at the initial optimal orientation and the new optimal orientation). The values of the mean firing activity are displayed in the histograms (on the right, $\mathrm{C}$ for control and A for adaptation).

(C) Orientation tuning curves of both neurons for control (solid lines) and post-adaptation (dashed lines) conditions, corresponding lighter colors represent error bars. Horizontal gray arrows show the direction of shifts of tuning curves and the black downward arrows represent the adapting orientation. The two neurons are tuned to $\mathrm{a} \pm 22^{\circ}$ similar oriented stimulus. (D) Shift-corrected crosscorrelograms of both neurons for their similar original optimal and new optimal gratings (left: control, right: post-adaptation).

Fig. 2 Correlation of peaks-strengths pre- and post-adaptation. (A) Linear regression analyses of the peaks-strengths for the original optimal orientation (left, 87 significant cases found, $\mathrm{n}=105$ ) and for the new acquired optimal orientation (right, 75 significant cases found, $\mathrm{n}=105)$. The dotted line indicates best-fit 45 degree line passing through the origin. $\mathrm{X}$-axis and $\mathrm{Y}$-axis show the peaks-strengths pre- and post-adaptation respectively. Solid black line in each graph displays the linear regression trend $\left(\mathrm{R}^{2}=0.17\right.$ for the original optimal orientation, $\mathrm{R}^{2}=8 \cdot 10^{-5}$ for the new optimal orientation). (B) Total distribution of peaks-strengths for all cell-pairs, green dots and curves represent preadaptation condition, orange dots and curves show post-adaptation condition. Results for the original optimal orientation are on the left and for new optimal orientation on the 
right. Below are illustrated the respective non-linear regression curves (original optimal: $\mathrm{R}^{2}$ control $=0.99, \mathrm{R}^{2}$ post-adaptation $=0.99$, new optimal: $\mathrm{R}^{2}$ control $=0.97, \mathrm{R}^{2}$ post-adaptation $\left.=0.97\right)$.

(C) Difference of connectivity strength between closely tuned neurons (gray bars) and differently tuned neurons (black bars) for control and post-adaptation. The peak-strength is significantly higher for closely tuned neurons in both conditions. (D) Averaged peakstrength at all orientations of interest (original and new optimal orientations on the left, adapting orientation on the right). The red dashed line represents the mean peak-strength (0.022) which is maintained post-adaptation.

Fig. 3 Population analyses of the modulation of peaks-strengths and spontaneous activity. (A) Cases of diminution of the mean peaks-strengths post-adaptation for the original optimal orientation (left) and the new optimal orientation (right), significant declines of 0.018 (two-tailed t-test, $p<0.05$ ) and 0.019 (two-tailed t-test, $p<0.05$ ) were observed for both orientations respectively. (B) Cases of augmentation of the mean peaks-strengths following adaptation for both orientations (original and new optimal), the increase of mean peak-strength for the new optimal orientation is almost $50 \%$ superior to the increase for the original optimal ( 0.027 and 0.015 respectively). (One-tailed unpaired ttest, 95\% significance level, $p=0.03$ ). (C) Left: example of CCG's between spike trains of two neurons. The peak magnitudes were $0.06,0.013$ and 0.061 for control, postadaptation and recovery (one hour after adaptation process), respectively. Right, upper: other examples of correlation variability for these three conditions. Right, lower: random sample test of averaged peaks strengths $(n=41)$ between control, post-adaptation and recovery period. Error bars in the histogram indicate the standard error of the mean. The observed peaks were $0.022 \pm 0.003,0.019 \pm 0.002,0.022 \pm 0.003$, respectively. (D) Averaged peak-strength (control and post-adaptation) for two conditions: during visual stimulation (red histograms) and during spontaneous activity (black histograms). 
Significant difference was observed for both conditions. (E) Example of two CCG's at two different times (two attempts separated by one hour). This example shows the stability of the peak-strength over a period of time.

Fig. 4 Variability of firing-responses. (A) Fano factors were calculated by dividing the variance $\left(\sigma^{2}\right)$ by the mean firing rate $(\mu)$ of every neuron from each pair at original (left) and new optimal (right) orientations. This factor increased from 0.49 to 0.57 for the original preferred orientation (paired two-tailed t-test, $p<0.05$ ) and decreased from 0.58 to 0.48 for the new preferred orientation (paired two-tailed t-test, $p<0.05$ ). (B) Gaussian orientation tuning curves plotted from raw data of two neurons. (C) Probabilities values within each bin $(7 \mathrm{~ms})$ during the stimulus period of presentation $(4.1 \mathrm{~s})$ for the same neurons. We observed a large decorrelation for the original orientation $\left(\mathrm{R}^{2}\right.$ pre-adaptation $=$ $0.6, \mathrm{R}^{2}$ post-adaptation $=0.09$, top) whereas the correlation is stable for the new optimal orientation $\left(\mathrm{R}^{2}\right.$ pre-adaptation $=0.5, \mathrm{R}^{2}$ post-adaptation $=0.8$, bottom $)$.

Fig. 5 Relationship to neuronal behavior. (A) Differences of peak-strength pre- and postadaptation between attracted and retracted neurons at the original optimal orientation. Histograms on the right side represent the average. The results demonstrate significant differences between attracted and retracted neurons (higher peak-strength for attracted neurons). (B and C) Similar to A for new optimal orientation and adapting orientation, respectively.

Fig. 6 Spike wave-forms and tuning bandwidth. (A) Separation of spikes based on trough-to-peak time, regular spikes had a trough-to-peak time greater than $0.5 \mathrm{~ms}$ (blue dots) and fast spikes had a smaller value (inferior to $0.5 \mathrm{~ms}$, green dots). (B) Example of 
two neurons with two different spike-shapes (blue unit: regular-spiking cell, green unit: fast-spiking cell). (C) Peak-strength modulation of all possible groups (RS-RS: blue plots, FS-FS: green plots, RS-FS: gray plots). No significant difference was observed between these spike-types. (D) Connection probability and tuning bandwidth. Tuning bandwidths measured (full width at half magnitude) for the original and the new optimal orientations. Neuronal pairs were classified in relation to the augmentation (green dots) or the diminution (red dots) of the peaks-strengths post-adaptation. The higher decrease of bandwidth is occurring for new optimal orientation $\left(26.4^{\circ}\right.$ to $17.3^{\circ}$, Mann-Whitney test, 95\% significance level, $\mathrm{p}<0.05)$.

Fig. 7 Cell-assembly computations. (A) Orientation tuning curves and Gaussian fits of five neurons (respective waveforms shown on the left) recorded simultaneously and responding preferentially to $45^{\circ}$ grating before adaptation. All neurons shifted their original optimal orientation to reach $90^{\circ}$ grating (corresponding to the adapter, downward black arrow in the plots), error bars in the tuning curves indicate standard error of the mean. (B) Cluster separation of all five neurons using the Trial-count correlation (TC), scatter plots are displayed for each neuronal pair of the entire group, mean TC's values are indicated by the horizontal bars. (C) Connectivity matrices of peak-strengths for original optimal orientation at control condition (top left) and post-adaptation (top right), and for the new optimal orientation at control (bottom left) and post-adaptation (bottom right). Colored scale indicates the normalized peaks-strengths. (D) Putative connections plotted from the matrices indicating the strength of connectivity (thickness of projections); red lines indicate newly formed connections after adaptation. (E) Comparative histograms of averaged peaks-strengths for the entire matrices between both conditions for original optimal orientation (top) and new optimal orientation (bottom). (F) 
Example of an orientation-map generated using optical imaging. It shows the changes of the entire map post-adaptation.

Fig. 8 Visual stimulation, cell separation and crosscorrelation. (A) Time course stimulus. Left: control condition, 8 orientations presented in random order within the receptive fields of recorded neurons in 25 trials (4.1 s each trial, 1-3 s interval time). Middle: one grating (non-preferred orientation in control) is presented continuously (12 min). Right: post-adaptation condition, all orientations are projected as in the control step. (B) Trialcount correlation (TC). Upper left side: TC values for all trials $(n=1894)$ accumulated over all selected neuron-pairs. Downer left side: Averaged TC values for each cell pair $(\mathrm{TC}=-0.005)$. Right side: Histogram-distribution of the averaged TC for each neuronal pair, gray curve represents the normal distribution curve. (C) Histological brain slice showing the electrode penetration and the recording site. (D) Examples of crosscorrelogram analyses indicating the $\pm 5 \mathrm{~ms}$ time range (time-delay of putative direct synaptic connections between cells). Red line indicates the $95 \%$ statistical threshold for the bin's significance; Y-axis shows the peak-strength computed from the counts/bin. 
A
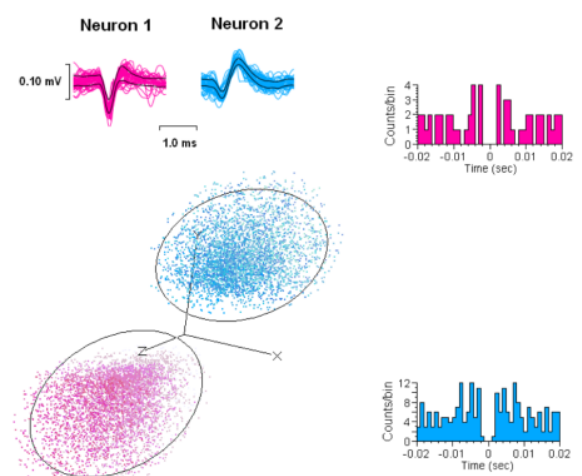

B
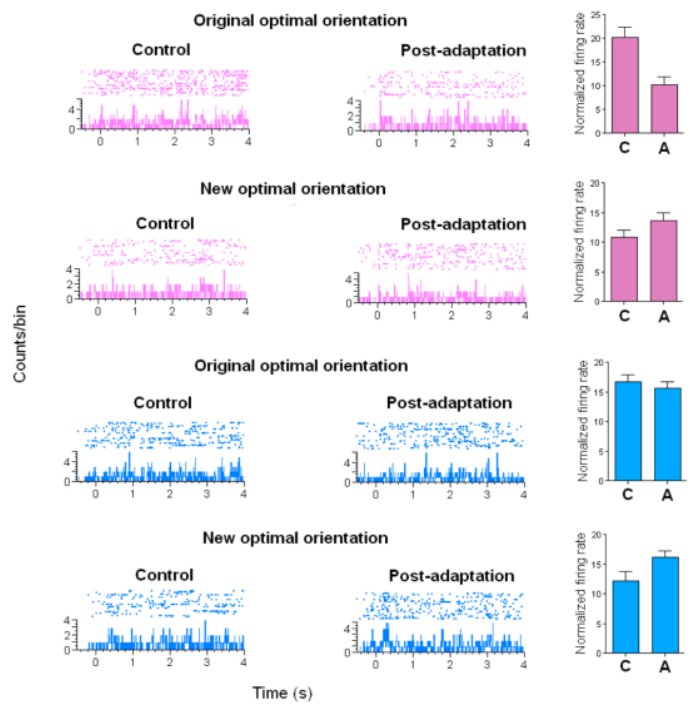

C

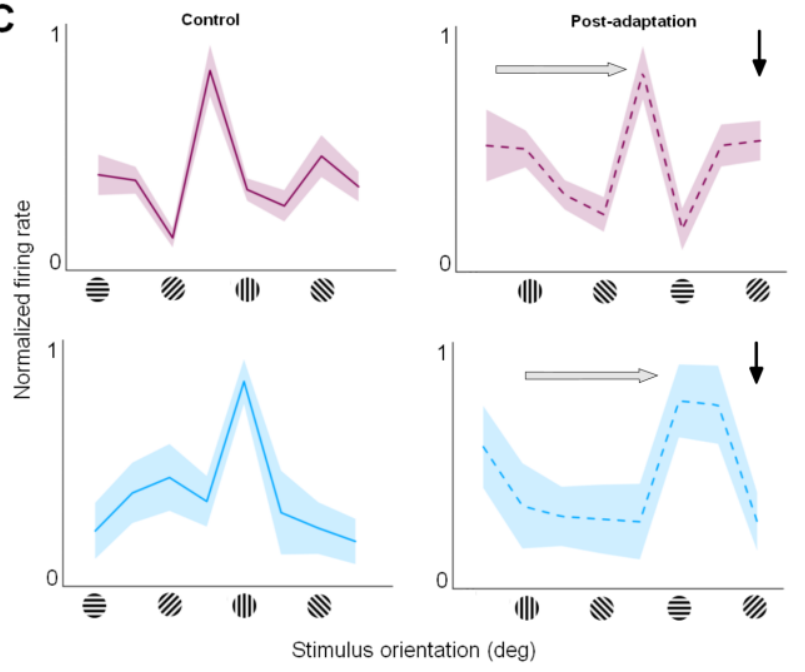

D
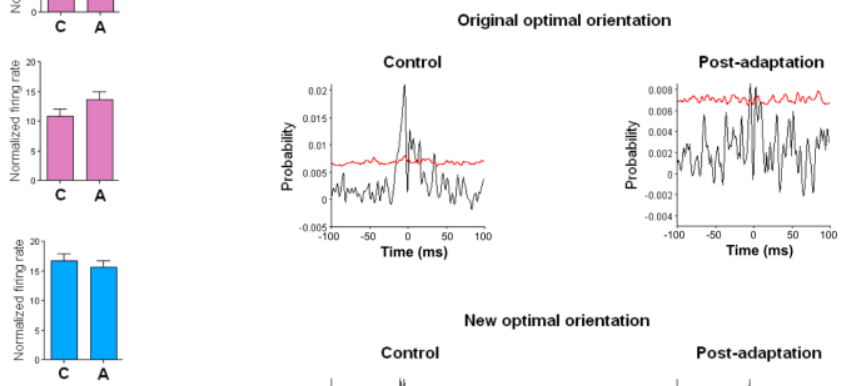
A
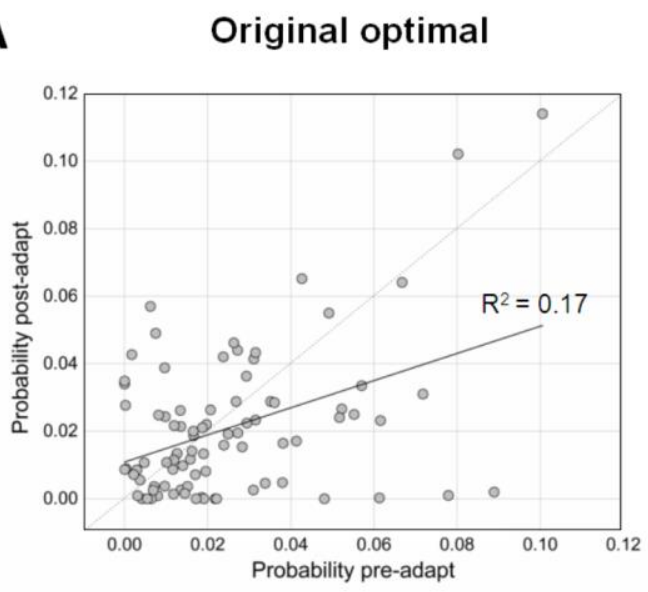

B
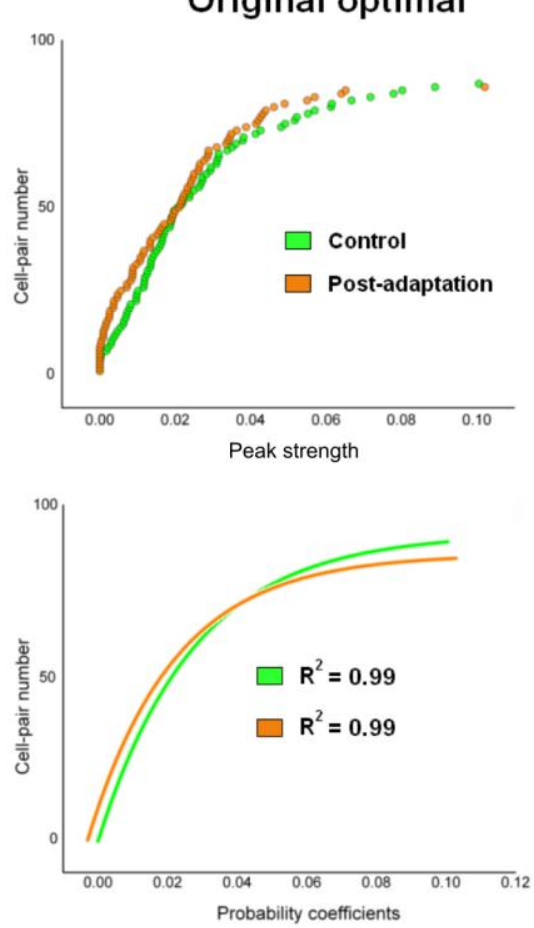

C

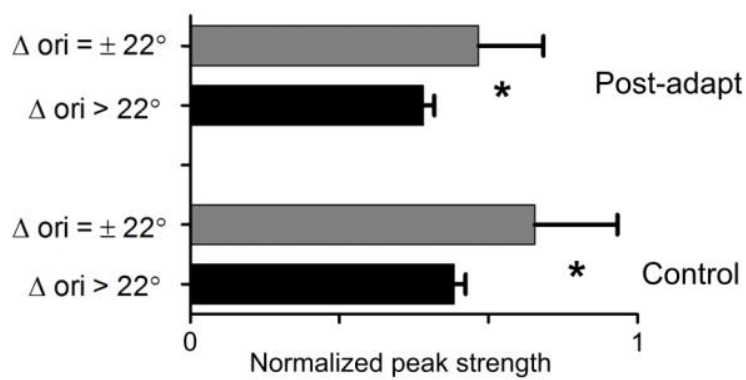

\section{New optimal}

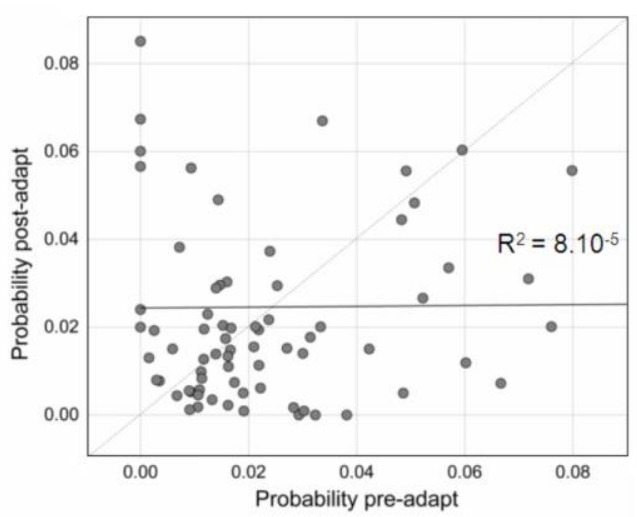

New optimal
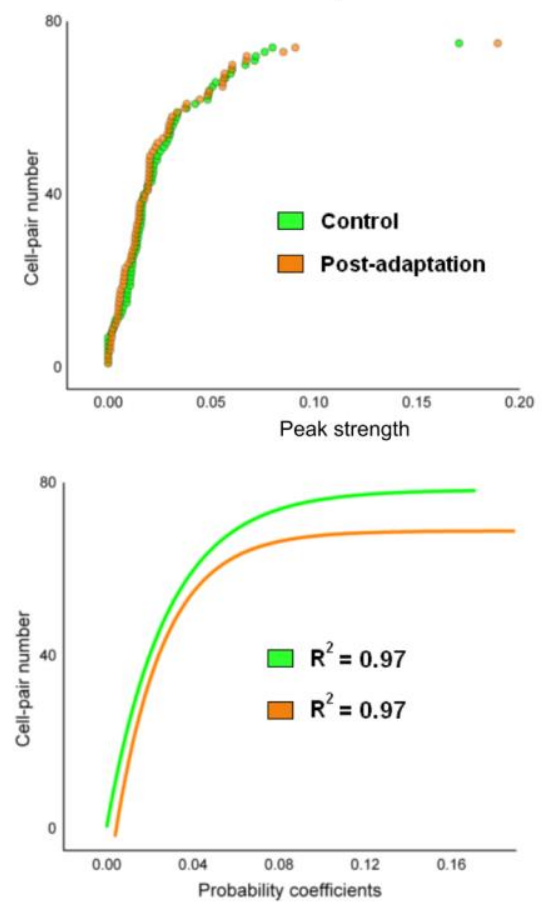

D

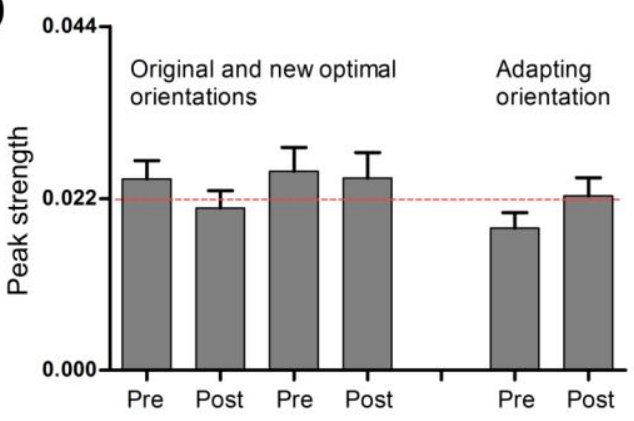


A

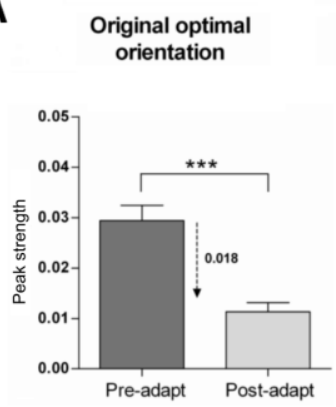

B

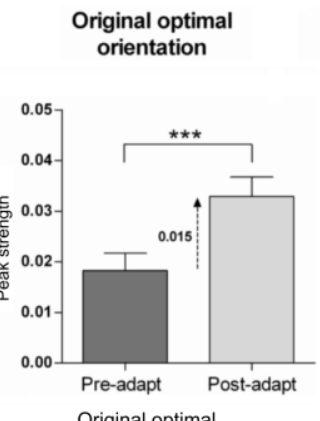

A

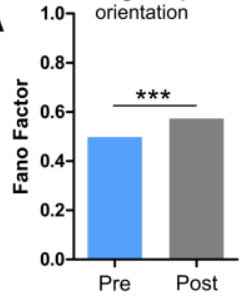

B
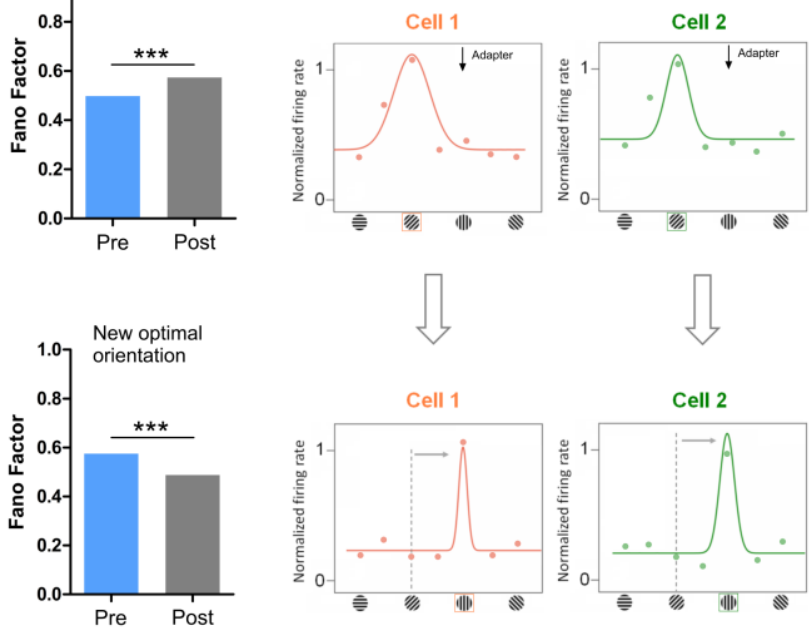

New optimal

orientation

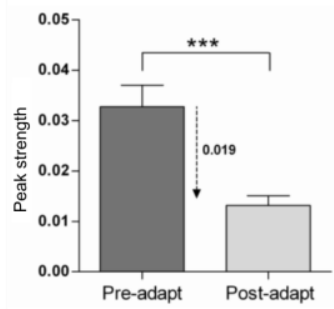

New optimal orientation

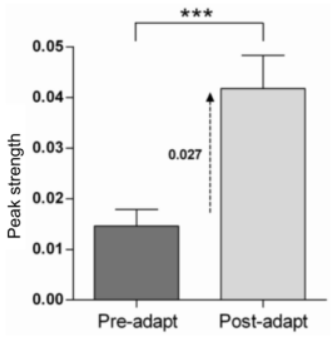

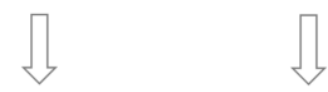

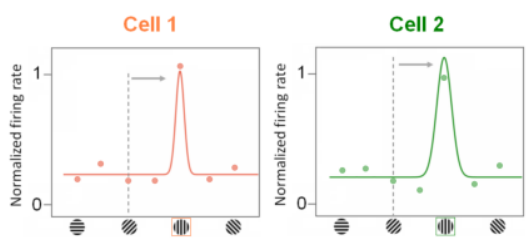

C

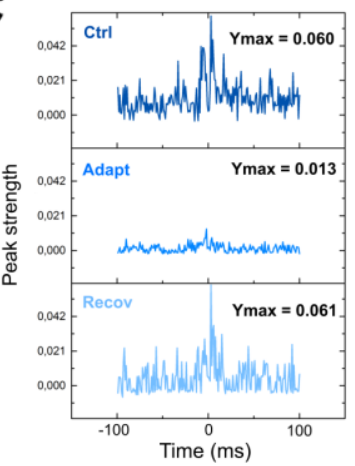

D

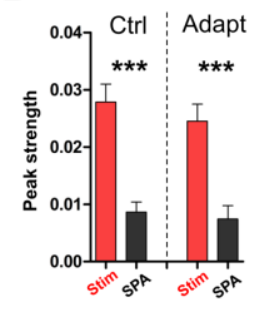

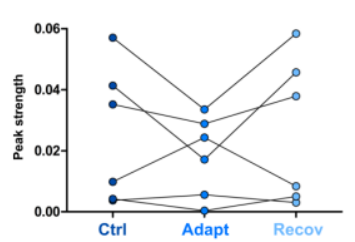

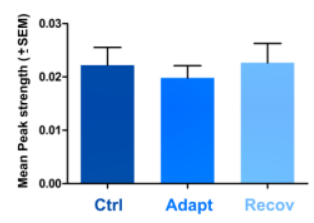

E

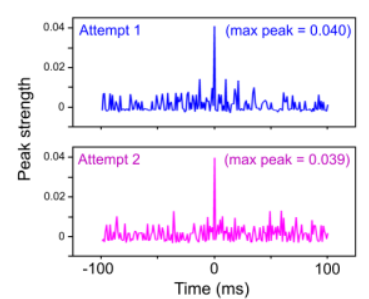

C $\quad$ OO Original optimal orientation
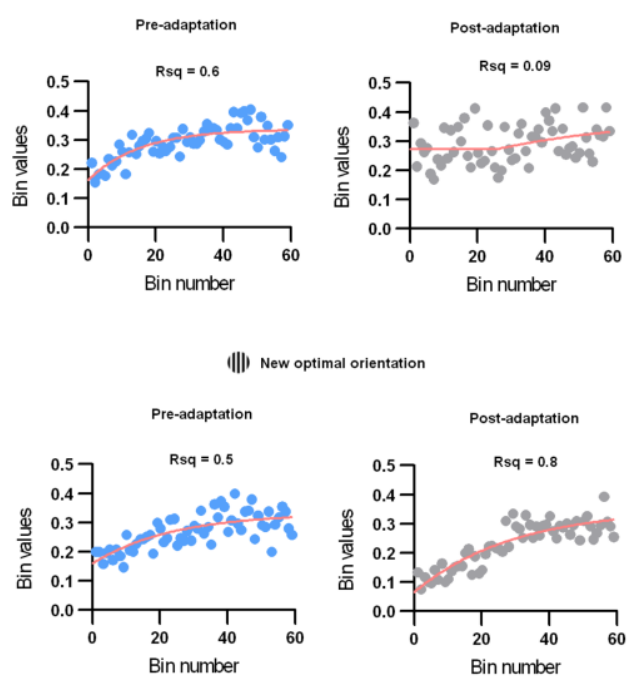


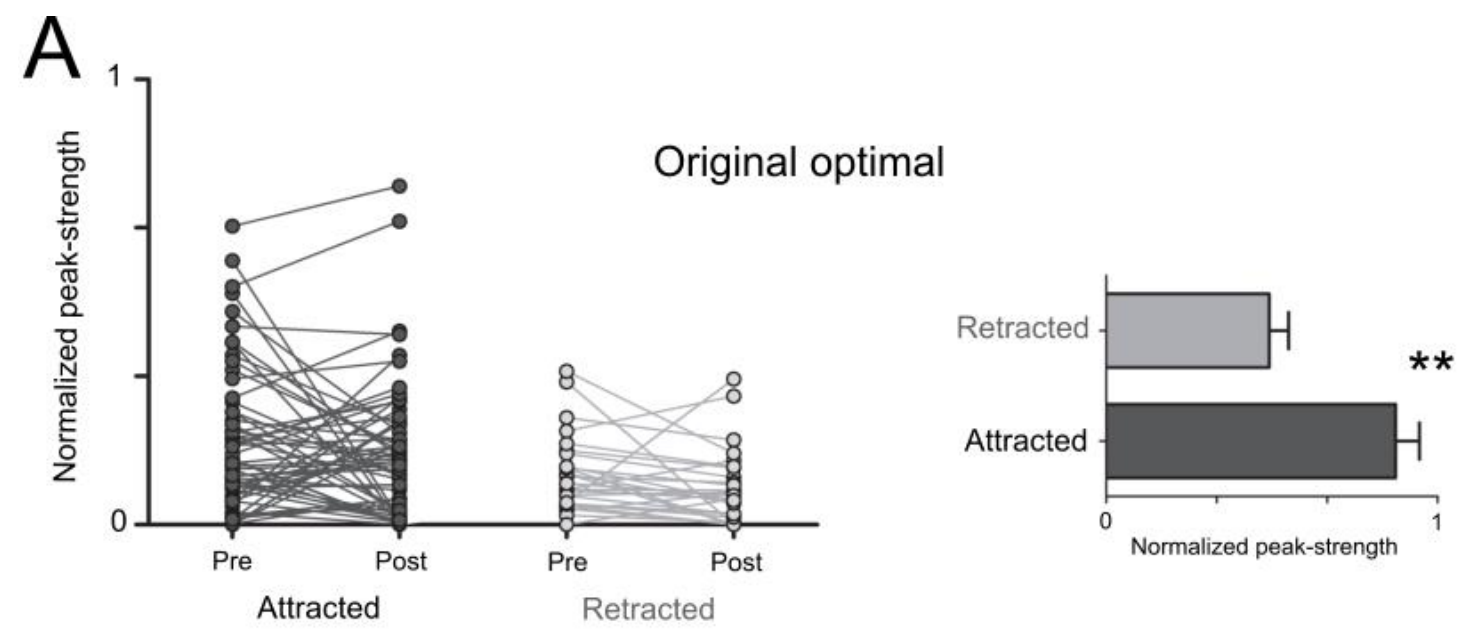

B
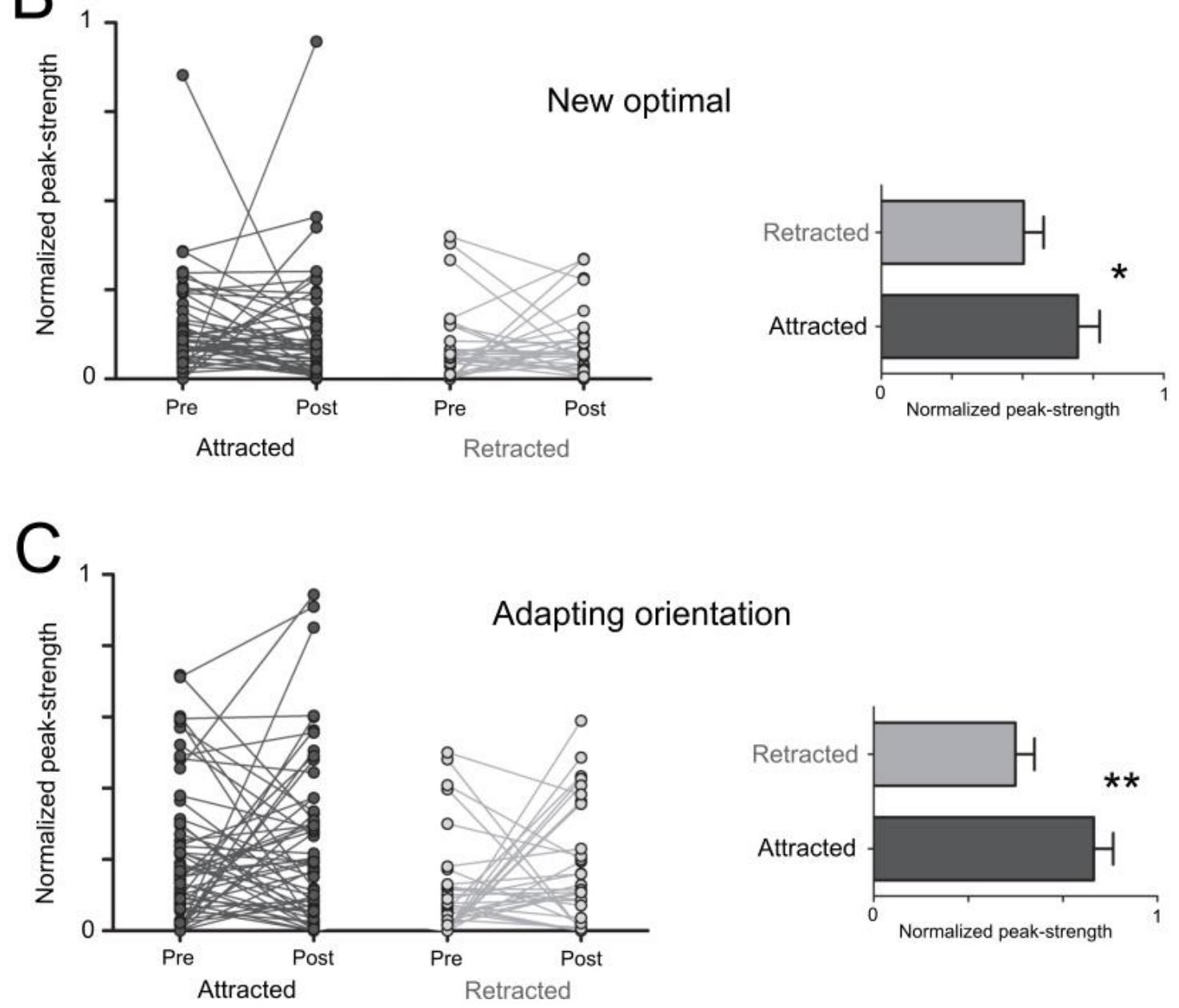
A

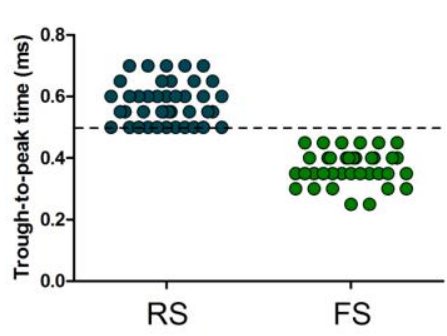

C

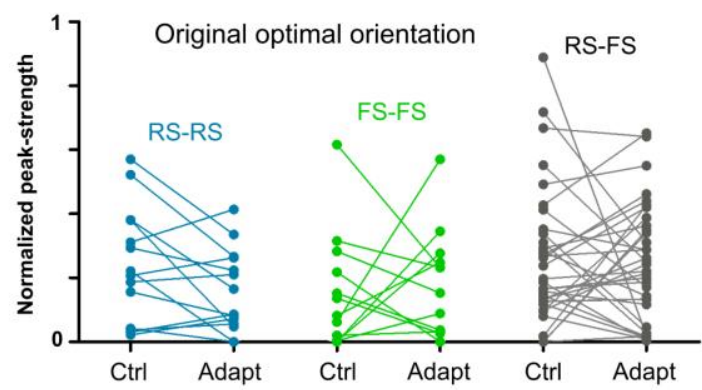

D

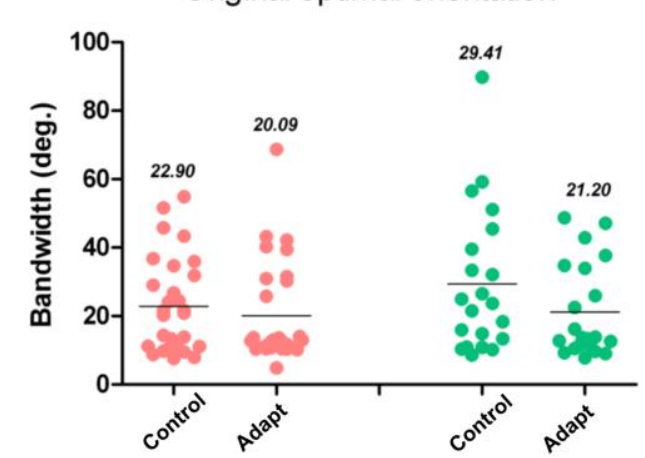

B
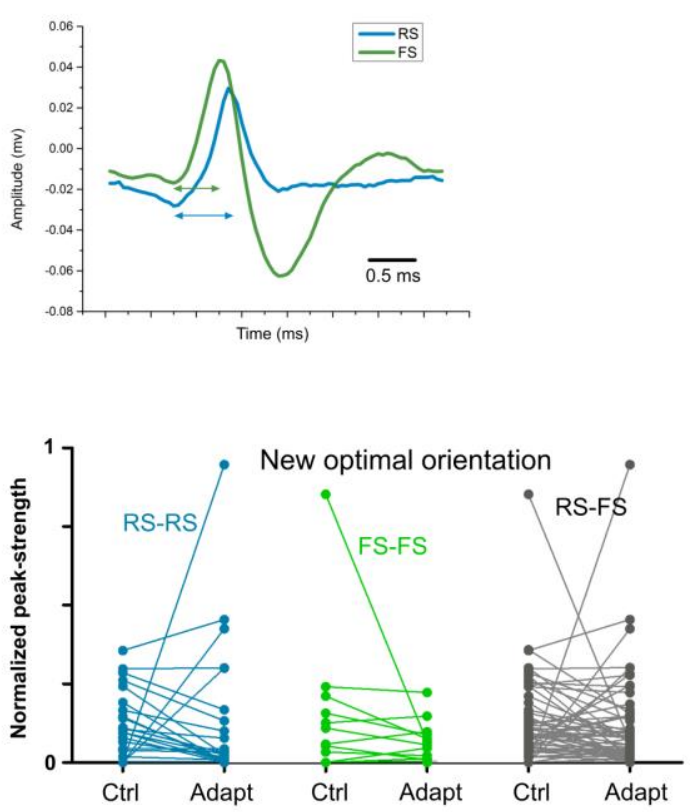

New optimal orientation

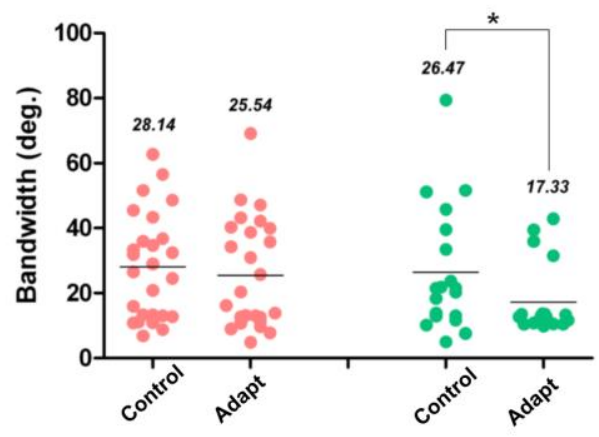

C

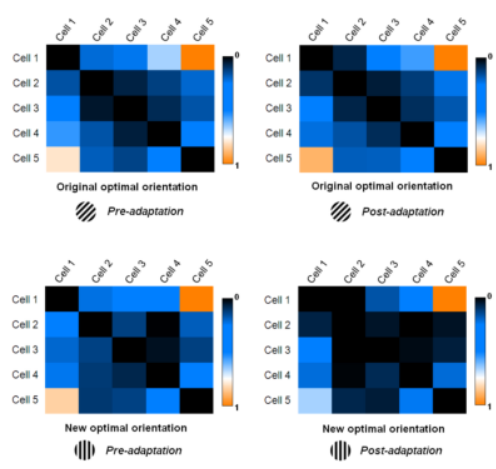

D

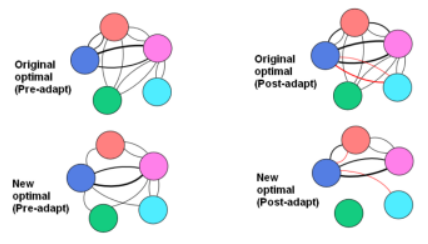

B

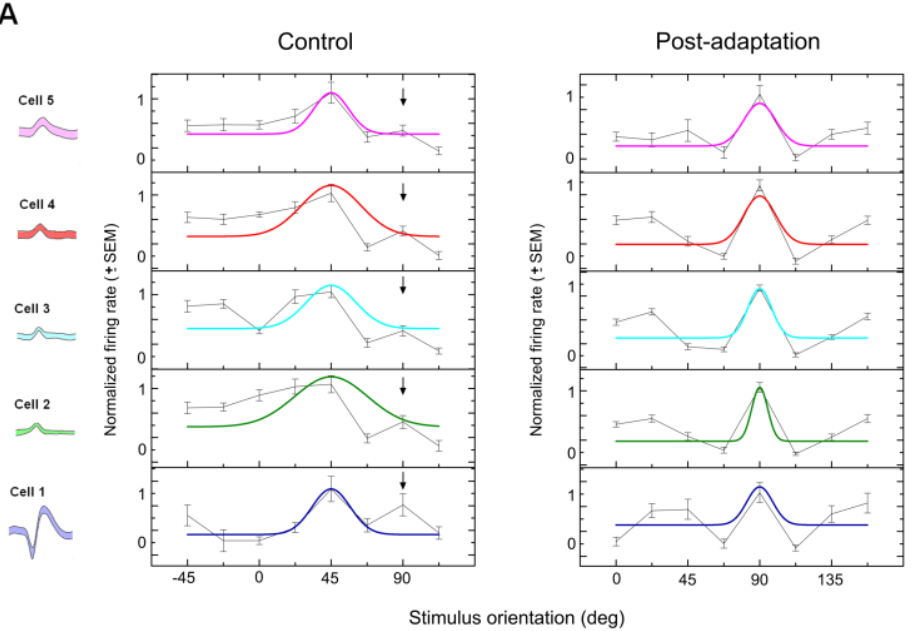

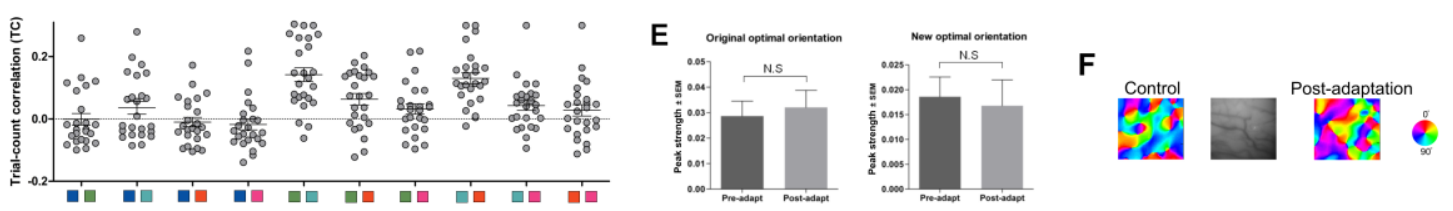


A

Control

Post-adaptation
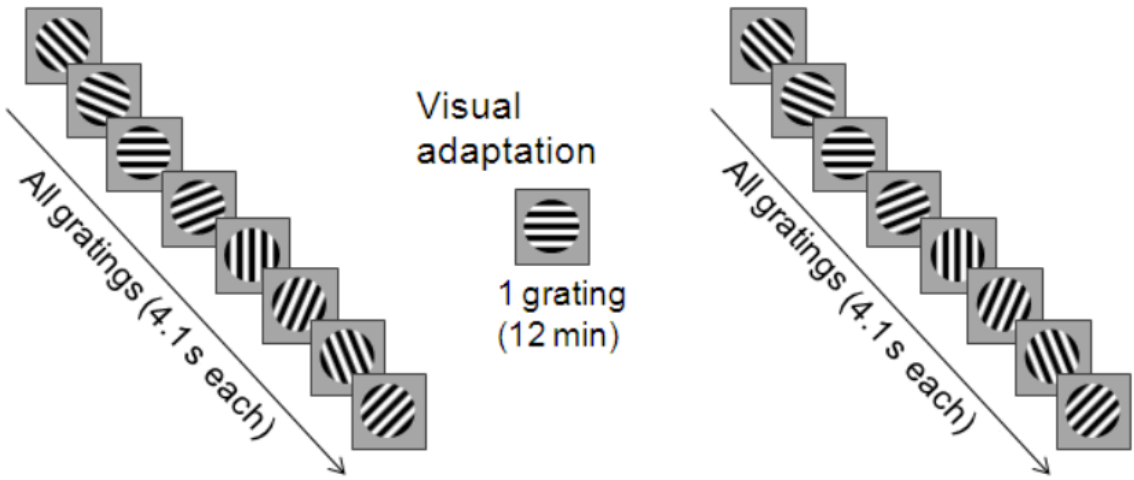

B
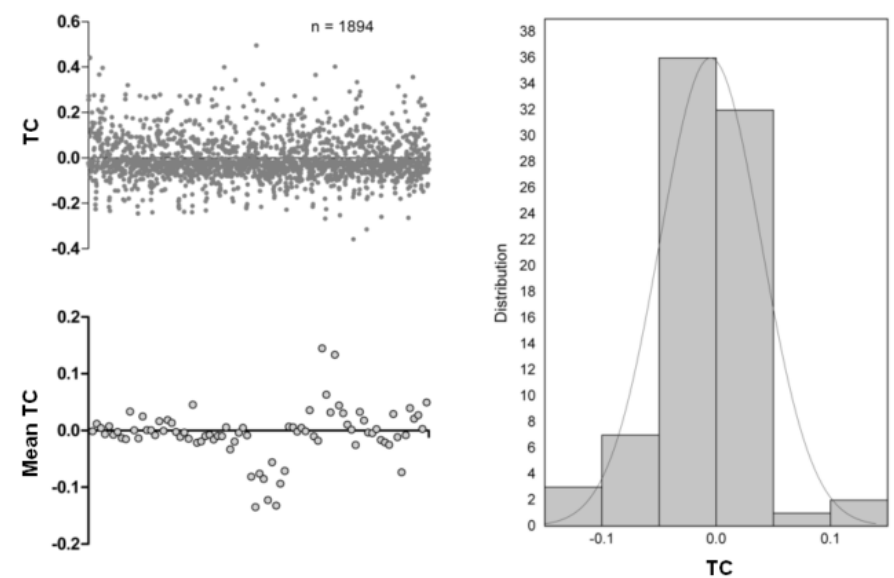

C

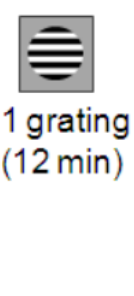

(12 min)

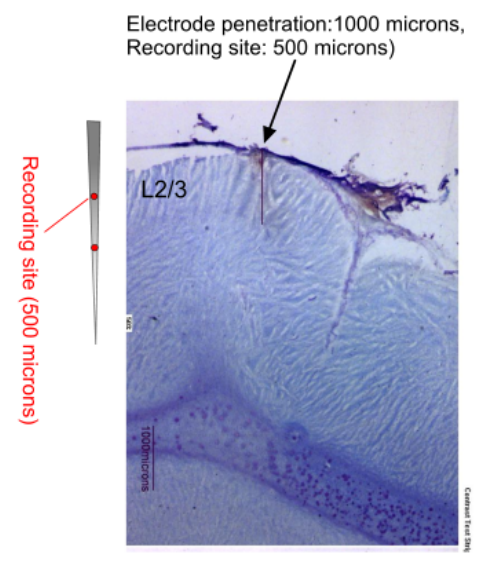

D

Control

5 ms time-window

Post-adaptation
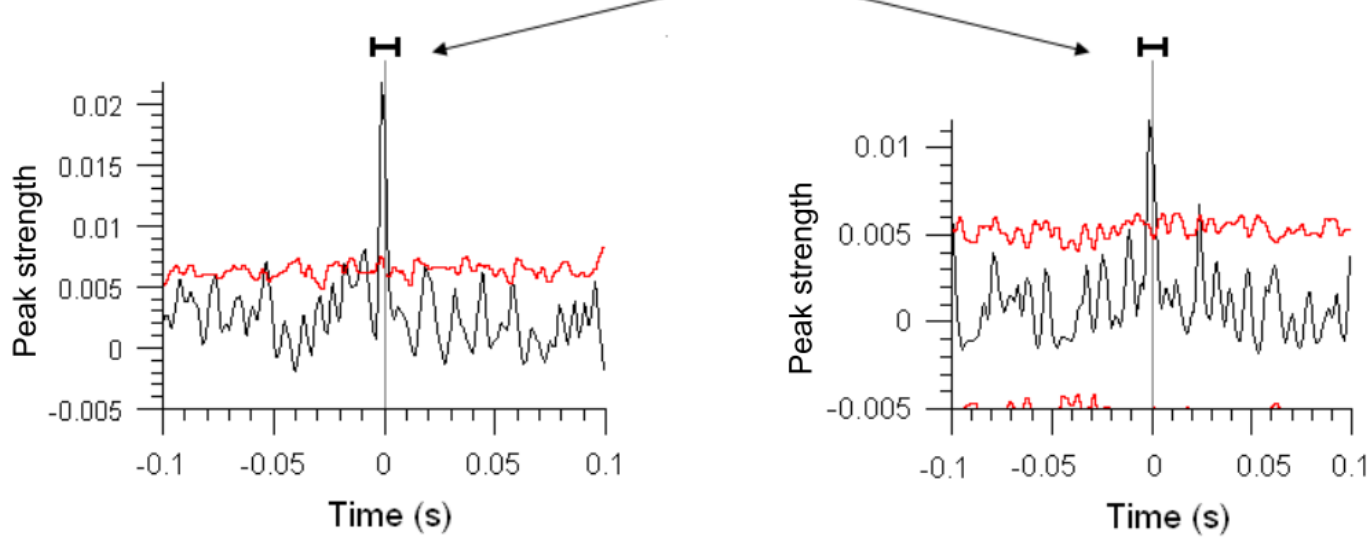